\title{
Area Specific Confidence Intervals for a Small Area Mean under the Fay-Herriot Model
}

\author{
Yegnanew A. Shiferaw, Jacqueline S. Galpin \\ School of Statistics and Actuarial Science, University of the Witwatersrand
}

\begin{abstract}
Small area estimates have received much attention from both private and public sectors due to the growing demand for effective planning of health services, apportioning of government funds and policy and decision making. Surveys are generally designed to give representative estimates at national or district level, but estimates of variables of interest are often also needed at lower levels. These cannot be reliably obtained from the survey data as the sample sizes at these levels are too small. Census data are often available, but only give limited information with respect to the variables of interest. This problem is addressed by using small area estimation techniques, which combine the estimates from the survey and census data sets. The main purpose of this paper is obtaining confidence intervals based on the empirical best linear unbiased predictor (EBLUP) estimates. One of the criticism of the mean squared error (MSE) estimators is that it is not area-specific since it does not involve the direct estimator in its expression. However, most of the confidence intervals in the literature are constructed based on those MSEs. In this paper, we propose area specific confidence intervals for small area parameters under the Fay-Herriot model using area specific MSEs. We extend these confidence intervals to the difference between two small area means. The effectiveness of the proposed methods are also investigated via simulation studies and compared with the Cox (1975) and Prasad and Prasad and Rao (1990) methods. Our simulation results show that the proposed methods have higher coverage probabilities. Those methods are applied to the percentage of food expenditure measures in Ethiopia using the 2010/11 Household Consumption Expenditure (HCE) survey and the 2007 census data sets.
\end{abstract}

Corresponding Author: Yegnanew A Shiferaw (yegnanews@uj.ac.za)

Jacqueline S Galpin (Jacqueline.Galpin@wits.ac.za). 
Keywords. Area specific, Basic area level model, EBLUP estimator, Confidence interval.

MSC: 62D05; 62F99.

\section{Introduction}

Small area statistics are needed for policy and decision making, local planning and fund distribution. It is known that direct estimates from a survey are highly unreliable as they have large standard errors, coefficients of variation and wide confidence intervals. The main reason behind this is that a sample survey is designed to provide reliable data for large areas such as national or regional levels, rather than that of small areas (Datta and Ghosh , 2012; Rao , 2003). Combining information from different surveys or from a census and a survey may address the problem (Chatterjee et al. , 2008; Rao , 2003). This is a less expensive tool for increasing sample sizes in order to improve the precision of the estimates.

In the Fay-Herriot model it is assumed that the parameter of interest is related to the vector of auxiliary variables through a linear regression model: $\theta_{i}=\mathbf{x}_{\mathbf{i}}^{\prime} \boldsymbol{\beta}+v_{i}, i=1, \ldots, m$. Let $y_{i}(i=1, \ldots, m)$ denote the direct small area estimator of $\theta_{i}$ for area $i$ from the survey; $\mathbf{x}_{\mathbf{i}}=\left(x_{1 i}, \ldots, x_{k i}\right)^{\prime}$ is a $k \times 1$ vector of known covariates for area $i$ related to the target parameters and $\beta=\left(\beta_{1}, \ldots, \beta_{k}\right)^{\prime}$ is a $k \times 1$ vector of regression coefficients. Using this, the Fay-Herriot model (Fay and Herriot, 1979) is defined as

$$
y_{i}=\mathbf{x}_{\mathbf{i}}^{\prime} \boldsymbol{\beta}+v_{i}+e_{i}, i=1, \ldots, m,
$$

where the area specific random effects $v_{i}$ are assumed to be independent and identically distributed (iid) with $E\left(v_{i}\right)=0, \operatorname{Var}\left(v_{i}\right)=A(\geq 0)$ and $e_{i} \stackrel{i i d}{\sim} N\left[0, \psi_{i}\right]$.

Small area means or totals can be expressed as linear combinations of fixed and random effects. The best known method for the prediction of mixed effects is the best linear unbiased predictor (BLUP). It is a weighted combination of the direct estimator $y_{i}$ and the regression synthetic estimator $\mathbf{x}_{i}^{\prime} \hat{\boldsymbol{\beta}}(\mathrm{Rao}, 2003)$. The BLUP for $\theta_{i}$ for the Fay-Herriot model is given by

$$
\tilde{\theta}_{i}^{B}=\left(1-\gamma_{i}\right) y_{i}+\gamma_{i} \mathbf{x}_{i}^{\prime} \tilde{\boldsymbol{\beta}}
$$

When the unknown parameters $(\beta, A)$ are replaced by their suitable estimators $(\hat{\beta}, \hat{A})$ obtained from different methods of estimation, for example, the method of moments (PR) used by Prasad and Rao (1990), Maximum Likelihood (ML) and Restricted Maximum Likelihood (REML) used by Datta and Lahiri (2000), the 
Fay and Herriot (1979) (FH) estimation method, we obtain the empirical best linear unbiased predictor (EBLUP) of $\theta_{i}$ which is given by

$$
\hat{\theta}_{i}^{E B}=\left(1-\hat{\gamma}_{i}\right) y_{i}+\hat{\gamma}_{i} \mathbf{x}_{\mathbf{i}}^{\prime} \hat{\beta}, \quad i=1, \ldots, m,
$$

where $\hat{\boldsymbol{\beta}}=\left(X^{\prime} \hat{\mathbf{V}}^{-1} X\right)^{-1} X^{\prime} \hat{\mathbf{V}}^{-1} y, \hat{\gamma}_{i}=\frac{\psi_{i}}{\psi_{i}+\hat{A}}$ and $\hat{\mathbf{V}}=\operatorname{diag}\left(\psi_{1}, \ldots, \psi_{m}\right)+\hat{A} \mathbf{I}_{m}$.

Using the EBLUP estimate from a small area model, one can obtain confidence interval (CI), which is the area of interest for this paper. Datta et al. (2002) and Diao et al. (2013) among others have derived CIs using Taylor series expansion method. Hall and Maiti (2006), Chatterjee et al. (2008) and Kubokawa and Nagashima (2012) also addressed it using the parametric bootstrap method. This shows that resampling methods and Taylor series methods are common approaches for estimating MSEs and developing CIs when exact formulas are not available.

Calibration and computational difficulties of bootstrap methods have been discussed by Hall et al. (2000) and Nankervis (2005). In addition to this, the coverage accuracy of CIs based on bootstrap method has been improved through calibration (Chatterjee et al. ,2008). Chatterjee et al. (2008, page 7) state that "however, it is not always clear what property of an interval, that is, length, coverage, end points or some other characteristic, ought to be calibrated". "In addition to the computational difficulties especially in the case of calibration, these methods have other issues, for example, choosing between equal tail or shortest interval quantile points, and so on" (Diao et al. (2013, page 498)). There are also questions on the use of pivotal statistics and calibration (Chatterjee et al. , 2008). Considering this, it is found that calibration is both time and computational effort consuming and the results often lack straightforward interpretability (Chatterjee et al. ,2008). It is also not clear on how to construct CIs for the difference of two small area means using resampling approach (Diao et al. , 2013).

However, CIs based on the Taylor series expansion method are easy to implement in practice. This is because the model parameters need to be estimated only once using standard software, and can then be used in the construction of plug-in type CIs (Diao et al. ,2013).

One of the criticism of the MSE estimators are that they are not area-specific in the sense that they do not explicitly depend on the direct estimator although the area-specific auxiliary data is involved in the component of the uncertainty due to estimation of the regression coefficient (Rao ,2003). Following Rao (2001), we propose area specific MSE estimators and use them to construct CIs. It is known that most of the CIs in the literature are constructed by using the non area specific MSEs. Having this in mind, the objective of this paper is to propose area specific confidence intervals for a small area mean based on the EBLUP 
estimator in the Fay-Herriot model.

It should be noted that when the estimated variance of the random component is negative, Prasad and Rao (1990) set it equal to zero. Those zero estimates will cause problems in the computing procedure and also lead the EBLUP estimator to run into difficulties (Chatterjee and Lahiri, 2002; Li , 2006). In addition, the contribution of the MSE estimate, assuming all parameters are known, becomes zero. This is one of the limitation of CIs based on EBLUP.

In summary, construction of CIs has received little attention when compared to point estimation in the issue of small area estimation methodology. Construction of CIs has also been limited and restricted to individual small area means. Furthermore, interval estimation for two or more population means is still an area to which very little attention has been paid. Most recently, Diao et al. (2013) proposed CIs for a small area mean as well as for the difference of two small area means. "We note that, since the small area estimators are not independent, this extension from a single prediction CI to one for differences (or more generally, linear combinations) of predictions is not immediate" (Diao et al. ,2013, page 4). In this paper we proposed CIs for a small area mean and for the difference of two small area means based on EBLUP for a coverage accuracy of order $O\left(m^{-3 / 2}\right)$ under unequal sampling variances for the FH model.

This paper is organized as follows. In Section 2, we describe the MSE estimation. In Section 3, we present the proposed confidence intervals. In Section 4, we use the 2010/11 HCE survey and the 2007 census data sets to estimate percentage of food expenditure measures. The final Section presents concluding remarks.

\section{Mean Squared Error Estimation}

The most common practical problem in small area estimation is measuring the variability associated with the EBLUP. The MSE is generally used as a measure of variability under the EBLUP estimator. Methods of approximating and estimating $\operatorname{MSE}\left(\hat{\theta}_{i}^{\mathrm{EB}}\right)$ that includes the uncertainty when estimating $\hat{\boldsymbol{\beta}}$ and $\hat{A}$ have been studied extensively in the literature, e.g., in Rao (2003); Chatterjee et al. (2008); Datta and Ghosh (2012); Hall and Maiti (2006).

\subsection{Estimation of $\operatorname{MSE}\left(\hat{\theta}_{i}^{\mathrm{EB}}\right)$}

The MSE estimator based on the PR moment estimator is given by

$$
\widehat{\operatorname{MSE}}\left(\hat{\theta}_{i}^{\mathrm{EB}}\right)=g_{1 i}(\hat{A})+g_{2 i}(\hat{A})+2 g_{3 i}(\hat{A}),
$$


where $g_{1 i}(\hat{A})=\hat{A} \psi_{i}\left(\hat{A}+\psi_{i}\right)^{-1}, g_{2 i}(\hat{A})=\frac{\psi_{i}^{2}}{\left(\hat{A}+\psi_{i}\right)^{2}} \mathbf{x}_{i}^{\prime}\left\{\sum_{u=1}^{m}\left(\hat{A}+\psi_{u}\right)^{-1} \mathbf{x}_{u} \mathbf{x}_{u}^{\prime}\right\}^{-1} \mathbf{x}_{i}$, and $g_{3 i}(\hat{A})=2 \psi_{i}^{2}\left(\psi_{i}+\hat{A}\right)^{-3} m^{-2} \sum_{u=1}^{m}\left(\psi_{u}+\hat{A}\right)^{2}$.

Datta et al. (2005) obtained the MSE estimator based on the FH moment estimator which is given by

$$
\widehat{\operatorname{MSE}}\left(\hat{\theta}_{i}^{\mathrm{EB}}\right)=g_{1 i}(\hat{A})+g_{2 i}(\hat{A})+2 g_{3 i}(\hat{A})-b_{\hat{A}}(\hat{A})\left\{B_{i}(\hat{A})\right\}^{2},
$$

where $g_{3 i}(\hat{A})=2 m \psi_{i}^{2}\left(\psi_{i}+\hat{A}\right)^{-3}\left\{\sum_{u=1}^{m}\left(\psi_{u}+\hat{A}\right)^{-1}\right\}^{-2}$.

For the $\mathrm{FH}$ estimator $\hat{A}$, we need the following expression for its bias to terms of order $O\left(m^{-1}\right)$ :

$$
b_{\hat{A}}(\hat{A})=\frac{2\left[m \sum_{i=1}^{m}\left(\hat{A}+\psi_{i}\right)^{-2}-\left\{\sum_{i=1}^{m}\left(\hat{A}+\psi_{i}\right)^{-1}\right\}^{2}\right]}{\left\{\sum_{i=1}^{m}\left(\hat{A}+\psi_{i}\right)^{-1}\right\}^{3}} .
$$

Note that if $E\left[\widehat{\operatorname{MSE}}\left(\hat{\theta}_{i}^{\mathrm{EB}}\right)-\operatorname{MSE}\left(\hat{\theta}_{i}^{\mathrm{EB}}\right)\right]=o\left(m^{-1}\right)$, then $\widehat{\operatorname{MSE}}\left(\hat{\theta}_{i}^{\mathrm{EB}}\right)$ is the secondorder unbiased estimator.

\subsection{Area Specific MSE Estimation for EBLUP}

In this paper, we proposed alternative area specific MSE estimators by extending the Rao (2001) area specific estimators. Thus an area specific estimator of $g_{3 i}(A)$ is simply obtained by writing $g_{3 i}(A)$ as

$$
g_{3 i}^{*}\left(\hat{A}, y_{i}\right)=\psi_{i}^{2}\left(\psi_{i}+\hat{A}\right)^{-4} E\left(y_{i}-\mathbf{x}_{i}^{\prime} \hat{\boldsymbol{\beta}}\right)^{2} V(\hat{A}),
$$

where the middle expectation term can be estimated by $\left(y_{i}-\mathbf{x}_{i}^{\prime} \hat{\boldsymbol{\beta}}\right)^{2}$, as noted by Rao (2001). The resulting estimator $g_{3 i}^{*}(\hat{A})$ is area-specific since it involves the direct estimator. It is less stable than the Prasad and Rao $g_{3 i}(\hat{A})$ even if its instability will not affect the corresponding MSE estimator. As a result, the coefficient of variation $(\mathrm{CV})$ of $\widehat{\mathrm{MSE}}^{*}\left(\hat{\theta}_{i}^{\mathrm{EB}}\right)$ should be comparable to the $\mathrm{CV}$ of $\widehat{\operatorname{MSE}}\left(\hat{\theta}_{i}^{\mathrm{EB}}\right)$, at least for moderate to large $m$ (Rao , 2003).

(a) An area specific estimator of $g_{3 i}(\hat{A})$ according to Rao (2001) is given by

$$
\operatorname{Rao}(2001): \quad g_{3 \operatorname{Rao} i}\left(\hat{A}, y_{i}\right)=\frac{\psi_{i}^{2}}{\left(\psi_{i}+A\right)^{4}}\left(y_{i}-\mathbf{x}_{i}^{\prime} \hat{\boldsymbol{\beta}}\right)^{2} V(\hat{A}) .
$$


(b) We propose another area specific estimator of $g_{3 i}(\hat{A})$ which is an extension of Rao (2001)'s area specific $g_{3 R a o i}\left(\hat{A}, y_{i}\right)$ and is given by

$$
\mathrm{JY}: \quad g_{3 \mathrm{YY} i}\left(\hat{A}, y_{i}\right)=\frac{\psi_{i}^{2}}{\left(\psi_{i}+A\right)^{4}} \frac{\left(y_{i}-\mathbf{x}_{i}^{\prime} \hat{\boldsymbol{\beta}}\right)^{2}}{\hat{A}+\psi_{i}-\mathbf{x}_{i}^{\prime}\left(\mathbf{X}^{\prime} \Sigma^{-1} \mathbf{X}\right)^{-1} \mathbf{x}_{i}} V(\hat{A}),
$$

where $\operatorname{Var}\left(y_{i}-\mathbf{x}_{i}^{\prime} \tilde{\boldsymbol{\beta}}\right)=A+\psi_{i}-\mathbf{x}_{i}^{\prime}\left(\mathbf{X}^{\prime} \Sigma^{-1} \mathbf{X}\right)^{-1} \mathbf{x}_{i}$. This proposal standardizes the residuals in order to make them scale free (Ananya , 2007).

(c) We propose another $g_{3 J Y 1 i}(A)$ which includes area dependent auxiliary variables as

$$
\text { JY1 : } \quad g_{3 J Y 1 i}(A)=g_{3 i}(A)-g_{5 i}(A),
$$

where $g_{5 i}(A)=\frac{g_{2 i}(A)}{\left(A+\psi_{i}\right)^{2}} V(\hat{A})$. Note that this estimator does not include $y_{i}$, but it includes the area specific auxiliary variables unlike the usual $g_{3 i}(A)$.

In this paper, we derive the theorems using Stein identity. Stein (1981) showed that

$$
E\left[h_{i}(y)\left(y_{i}-x_{i}^{\prime} \beta\right)\right]=\left(\psi_{i}+A\right) E\left[\frac{\partial h_{i}(y)}{\partial y_{i}}\right],
$$

where $h_{i}(y)$ is an absolutely continuous function. This identity is called Stein identity (Datta et al. , 2011). The following regularity conditions are used for deriving all the theorems.

- Assumption 1: The quantities $\mathbf{x}_{i}, A, \psi_{i}$ are bounded.

- Assumption 2: $v_{i}$ and $e_{i}$ are mutually independent with $v_{i} \stackrel{i n d}{\sim} N[0, A]$ and $e_{i} \stackrel{i n d}{\sim} N\left[0, \psi_{i}\right], i=1, \ldots, m$

- Assumption 3: $\hat{A}=\hat{A}(\mathbf{y})$ is an estimator of $A$ which satisfies

(i) they are even functions of $\mathbf{y}$, so that $\hat{A}(-\mathbf{y})=\hat{A}(\mathbf{y})$,

(ii) they are translation invariant functions, so that $\hat{A}(\mathbf{y}+\mathbf{X} d)=\hat{A}(\mathbf{y})$ for any $d \in R^{p}$ and for all $\mathbf{y}$.

- Assumption 4: $\max _{1 \leq i \leq m} \mathbf{x}_{i}^{\prime}\left(\mathbf{X}^{\prime} \mathbf{X}\right)^{-1} \mathbf{x}_{i} \rightarrow 0$ as $m \rightarrow \infty$, or

$$
\max _{1 \leq i \leq m} \mathbf{x}_{i}^{\prime}\left(\mathbf{X}^{\prime} \mathbf{X}\right)^{-1} \mathbf{x}_{i}=O\left(m^{-1}\right),
$$

(Prasad and Rao, 1990) where $\mathbf{x}_{i}$ is the $\mathbf{i}$-th row of $\mathbf{X}$. All conditions are very often assumed in the small area estimation literature.

Theorem 2.1. Under the assumptions 1, 2, 3 and 4,

$$
\operatorname{Elg}_{3 J Y 1 i}(\hat{A})-g_{3 \gamma Y 1 i}(A) \mid=o\left(m^{-1}\right)
$$

The proof of this theorem can be found in a Supplementary material. 


\subsubsection{Area Specific MSE Estimation Based on the ML or REML Estimators}

According to Rao (2003), the two alternative area specific estimators based on the ML Estimators (Datta and Lahiri ,2000) are given by

$$
\widehat{\operatorname{MSE}}_{1}^{*}\left(\hat{\theta}_{i}^{\mathrm{EB}}\right) \approx g_{1 i}(\hat{A})-b_{\hat{A}}(\hat{A}) \nabla g_{1 i}(\hat{A})+g_{2 i}(\hat{A})+2 g_{3 i}^{*}\left(\hat{A}, y_{i}\right)
$$

and

$$
\widehat{\operatorname{MSE}}_{2}^{*}\left(\hat{\theta}_{i}^{\mathrm{EB}}\right) \approx g_{1 i}(\hat{A})-b_{\hat{A}}(\hat{A}) \nabla g_{1 i}(\hat{A})+g_{2 i}(\hat{A})+g_{3 i}(\hat{A})+g_{3 i}^{*}\left(\hat{A}, y_{i}\right),
$$

where $b_{\hat{A}}(\hat{A})=\frac{-\operatorname{tr}\left\{\left[\sum_{i=1}^{m}\left(\hat{A}+\psi_{i}\right)^{-1} \mathbf{x}_{i} \mathbf{x}_{i}^{\prime}\right]^{-1}\left[\sum_{i=1}^{m}\left(\hat{A}+\psi_{i}\right)^{-2} \mathbf{x}_{i} \mathbf{x}_{i}^{\prime}\right]\right\}}{\sum_{i=1}^{m}\left(\hat{A}+\psi_{i}\right)^{-2}}$.

Similarly, the two alternative area specific estimators based on the REML Estimators (Datta and Lahiri , 2000) are given by

$$
\widehat{\operatorname{MSE}}_{1}^{*}\left(\hat{\theta}_{i}^{\mathrm{EB}}\right) \approx g_{1 i}(\hat{A})+g_{2 i}(\hat{A})+2 g_{3 i}^{*}\left(\hat{A}, y_{i}\right)
$$

and

$$
\widehat{\operatorname{MSE}}_{2}^{*}\left(\hat{\theta}_{i}^{\mathrm{EB}}\right) \approx g_{1 i}(\hat{A})+g_{2 i}(\hat{A})+g_{3 i}(\hat{A})+g_{3 i}^{*}\left(\hat{A}, y_{i}\right)
$$

\subsubsection{Area Specific MSE Estimation Based on the Method of Moments}

The two alternative area specific estimators based on the FH Estimator (Datta et al. ,2005) are given by

$$
\widehat{\operatorname{MSE}}_{1}^{*}\left(\hat{\theta}_{i}^{\mathrm{EB}}\right) \approx g_{1 i}(\hat{A})+g_{2 i}(\hat{A})+2 g_{3 i}^{*}\left(\hat{A}, y_{i}\right)-b_{\hat{A}}(\hat{A})\left\{B_{i}(\hat{A})\right\}^{2},
$$

and

$$
\widehat{\operatorname{MSE}}_{2}^{*}\left(\hat{\theta}_{i}^{\mathrm{EB}}\right) \approx g_{1 i}(\hat{A})+g_{2 i}(\hat{A})+g_{3 i}(\hat{A})+g_{3 i}^{*}\left(\hat{A}, y_{i}\right)-b_{\hat{A}}(\hat{A})\left\{B_{i}(\hat{A})\right\}^{2} .
$$

Note that $g_{3 i}^{*}\left(\hat{A}, y_{i}\right)$ can be an area specific estimator of $g_{3 i}(\hat{A})$. See Rao (2003) for the detailed discussion about the extension of the MSE estimation to an area specific MSE estimation.

\section{Area Specific Confidence Intervals under the Fay-Herriot Model}

\subsection{Area Specific Confidence Intervals for a Small Area Mean}

In this paper, we develop a closed form area specific confidence intervals for $\theta_{i}$ based on $\hat{\theta}_{i}^{\mathrm{EB}}$ under unequal sampling error variances and that coverage probability is correct to $O\left(\mathrm{~m}^{-3 / 2}\right)$. We derive the theorem similarly to Diao et al. (2013) using Taylor series expansion. 
Theorem 3.1. For any real $z>0$,

$$
P\left[\theta_{i} \epsilon \hat{\theta}_{i}^{\mathrm{EB}} \pm z \times \sqrt{\widehat{\operatorname{MSE}}\left(\hat{\theta}_{i}^{\mathrm{EB}}\right)}\right]=2 \Phi(z)-1-\frac{z \phi(z)}{4} \eta+O\left(m^{-3 / 2}\right),
$$

where $\eta=\left(z^{2}+1\right) \frac{\psi_{i}^{2}}{A^{2}\left(A+\psi_{i}\right)^{2}} V(\hat{A})$ will give an EBLUP CI that matches the nominal $C P$ to the order of $O\left(m^{-3 / 2}\right)$.

Note that the proof of this theorem can be found in the Supplementary material.

These CIs based on the EBLUP can be written as

$$
\hat{\theta}_{i}^{\mathrm{EB}} \pm t_{\alpha}^{*} \sqrt{\widehat{\mathrm{MSE}}\left(\hat{\theta}_{i}^{\mathrm{EB}}\right)},
$$

where $t_{\alpha}^{*}=z_{\alpha / 2}(1+h(\hat{A})), h(\hat{A})=\left(z_{\alpha / 2}^{2}+1\right) \frac{\psi_{i}^{2}}{8 \hat{A}^{2}\left(\hat{A}+\psi_{i}\right)^{2}} V(\hat{A})$.

With this in mind, the recent CIs by Diao et al. (2013) is given by

$$
\text { Diao et al. (2013) : } \quad \hat{\theta}_{i}^{\mathrm{EB}} \pm t_{\alpha} \sqrt{\widehat{\mathrm{MSE}}\left(\hat{\theta}_{i}^{\mathrm{EB}}\right)},
$$

where $t_{\alpha}=z_{\alpha / 2}+\frac{z_{\alpha / 2}^{3}+z_{\alpha / 2}}{8 h_{i}^{4}} \frac{g_{3 i} \psi_{i}^{2}}{\hat{A}+\psi_{i}}$, and $h_{i}^{2}=g_{1 i}+g_{2 i}$ for the PR and REML estimators.

Both (3.1) and (3.2) are constructed by using the non area specific MSEs which are given by (2.1) and (2.2). In addition, Theorem 3.1 is based on the usual non area specific $g_{3 i}(A)$. However, the following corollaries are obtained by using the proposed area specific MSEs which are given by (2.8-2.13). Once Theorem 3.1 is proved, Corollary 3.1, Corollary 3.2 and Corollary 3.3 can be easily obtained through replacing the usual $g_{3 i}(A)$ by area specific $g_{3 i}\left(A, y_{i}\right)$ 's given by $(2.4),(2.5)$ and (2.6), respectively. The corresponding area specific CIs are also given in (3.7), (3.8) and (3.9), respectively.

Corollary 3.1. The CP of $I^{\text {CEBRao }}(\hat{A})$ based on the Rao (2001) is written as

$$
P\left[\theta_{i} \in I^{\text {CEBRao }}(\hat{A})\right]=2 \Phi(z)-1-\frac{z \phi(z)}{4} \eta_{\text {Rao }}+O\left(m^{-3 / 2}\right),
$$

where $\eta_{\text {Rao }}=\left(z^{2}+1\right) \frac{\psi_{i}^{2}}{\hat{A}^{2}\left(\hat{A}+\psi_{i}\right)^{3}}\left(y_{i}-\mathbf{x}_{i}^{\prime} \hat{\boldsymbol{\beta}}\right)^{2} V(\hat{A})$.

Corollary 3.2. The CP of $I^{\mathrm{CEBJY}}(\hat{A})$ based on JY is written as

$$
P\left[\theta_{i} \in I^{\mathrm{CEBJY}}(\hat{A})\right]=2 \Phi(z)-1-\frac{z \phi(z)}{4} \eta_{\mathrm{JY}}+O\left(m^{-3 / 2}\right),
$$

where $\eta_{\mathrm{JY}}=\left(z^{2}+1\right) \frac{\psi_{i}^{2}}{\hat{A}^{2}\left(\hat{A}+\psi_{i}\right)^{3}} \frac{\left(y_{i}-\mathbf{x}_{i}^{\prime} \tilde{\boldsymbol{\beta}}\right)^{2}}{\hat{A}+\psi_{i}-\mathbf{x}_{i}^{\prime}\left(\mathbf{X}^{\prime} \mathbf{\Sigma}^{-1} \mathbf{X}\right)^{-1} \mathbf{x}_{i}} V(\hat{A})$. 
Corollary 3.3. The CP of $I^{\mathrm{CEBJY1}}(\hat{A})$ based on JY1 is written as

$$
P\left[\theta_{i} \in I^{\mathrm{CEBJY} 1}(\hat{A})\right]=2 \Phi(z)-1-\frac{z \phi(z)}{4} \eta_{\mathrm{JY} 1}+O\left(m^{-3 / 2}\right),
$$

where $\eta_{\mathrm{J} Y 1}=\left(z^{2}+1\right) \frac{\psi_{i}^{2}}{\hat{A}^{2}\left(\hat{A}+\psi_{i}\right)^{3}}\left(\hat{A}+\psi_{i}-\mathbf{x}_{i}^{\prime}\left(\mathbf{X}^{\prime} \Sigma^{-1} \mathbf{X}\right)^{-1} \mathbf{x}_{i}\right) V(\hat{A})$.

\subsubsection{Comparison of Confidence Intervals for a Small Area Mean: Simulation Study}

We consider the simulation set-up by Datta et al. (2005) with minor modifications. The sampling error, $e_{i}$, are generated from $N\left(0, \psi_{i}\right)$ for $\psi_{i}$ specified by the following three different variance patterns: pattern (I) $0.7,0.6,0.5,0.4,0.3$, pattern (II) $2,0.6,0.5,0.4,0.2$ and pattern (III) $4,0.6,0.5,0.4,0.1$. The random effects $v_{i}$ are generated from two different distributions, namely normal $N(0,1)$ and Laplace $(0,1)$ distributions. We generate 10,000 data sets from $y_{i}=v_{i}+e_{i},(i=1, \cdots, m)$. The small areas are divided into five equal sized groups, and the $\psi_{i}{ }^{\prime}$ s remain the same in each group. For the FH model with $\mathrm{m}=15,20,30,40,50,60,80$ and 100 areas and $A=1$, we have investigated the coverage probabilities and average lengths of the proposed methods. The coverage probability $(\mathrm{CP})$ and average length (AL) of confidence intervals can be expressed as

$$
C P_{i}=\sum_{i=1}^{m} \frac{\left|\theta_{i} \in C I_{i}\right|}{m}, \quad A L_{i}=\sum_{i=1}^{m} \frac{\mid \text { length of } C I_{i} \mid}{m},
$$

where $C I_{i}, \mathrm{i}=1, \ldots, \mathrm{m}$, is the confidence interval of a certain method.

The main goal of this paper is to develop a closed form improved CIs for a small area mean based on the EBLUP using the Taylor series expansion methods under the FH model. In this section, a simulation study is conducted to investigate the performance of the proposed methods with the Cox (1975) and Prasad and Rao (1990) type CIs. The performance measures are the CP and AL for the 95\% CIs. The results are based on 10000 simulations under the three different sampling variance patterns. We wrote an extension of SAS codes written by Li (2007). We also compared the results obtained by PR, FH, ML and REML estimators. Consider the following six different types of CIs.

- Cox (1975) suggested to generate the following CI:

$$
\text { Method I (Meth I) : } \hat{\theta}_{i}^{\mathrm{EB}} \pm z_{\alpha / 2} \psi_{i}^{1 / 2}\left(1-\hat{\gamma}_{i}\right)^{1 / 2} \text {. }
$$

- Prasad and Rao (1990) proposed a CI which is given by

$$
\text { Method II (Meth II) }: \hat{\theta}_{i}^{\mathrm{EB}} \pm z_{\alpha / 2} \sqrt{\widehat{\mathrm{MSE}}\left(\hat{\theta}_{i}^{\mathrm{EB}}\right)} \text {. }
$$


- The improved CI based on the EBLUP is given by

$$
\begin{aligned}
& \text { Method III (Meth III) : } \hat{\theta}_{i}^{\mathrm{EB}} \pm t_{\alpha}^{*} \sqrt{\widehat{\operatorname{MSE}}\left(\hat{\theta}_{i}^{\mathrm{EB}}\right),} \\
& \text { where } t_{\alpha}^{*}=z_{\alpha / 2}(1+h(\hat{A})), h(\hat{A})=\left(z_{\alpha / 2}^{2}+1\right) \frac{\psi_{i}^{2}}{8 \hat{A}^{2}\left(\hat{A}+\psi_{i}\right)^{2}} V(\hat{A}) .
\end{aligned}
$$

The corresponding area specific versions of (3.6) are written as

$$
\text { CIRao : } \hat{\theta}_{i}^{\mathrm{EB}} \pm t_{\mathrm{Rao}} \sqrt{\widehat{\mathrm{MSE}}\left(\hat{\theta}_{i}^{\mathrm{EB}}\right)},
$$

where $t_{\text {Rao }}=z_{\alpha / 2}+\left(z_{\alpha / 2}^{3}+z_{\alpha / 2}\right) \frac{\left(A+\psi_{i}\right) g_{3 R a a i}}{8 A^{2}}, \quad g_{3 R a o i}(\hat{A})=\frac{\psi_{i}^{2}}{\left(A+\psi_{i}\right)^{4}}\left(y_{i}-x_{i}^{\prime} \hat{\beta}\right)^{2} V(\hat{A})$ and

$$
\mathrm{CIJY}: \hat{\theta}_{i}^{\mathrm{EB}} \pm t_{\mathrm{JY}} \sqrt{\widehat{\mathrm{MSE}}\left(\hat{\theta}_{i}^{\mathrm{EB}}\right)}
$$

where

$$
t_{\mathrm{JY}}=z_{\alpha / 2}+\left(z_{\alpha / 2}^{3}+z_{\alpha / 2}\right) \frac{\left(A+\psi_{i}\right) g_{3 J Y i}}{8 A^{2}}
$$

and

$$
g_{3 \mathrm{JY} i}\left(\hat{A}, y_{i}\right)=\frac{\psi_{i}^{2}}{\left(\psi_{i}+\hat{A}\right)^{4}} \frac{\left(y_{i}-\mathbf{x}_{i}^{\prime} \tilde{\boldsymbol{\beta}}\right)^{2}}{\hat{A}+\psi_{i}-\mathbf{x}_{i}^{\prime}\left(\mathbf{X}^{\prime} \Sigma^{-1} \mathbf{X}\right)^{-1} \mathbf{x}_{i}} V(\hat{A})
$$

Another improved CI based on the EBLUP is written as

$$
\mathrm{CIJY} 1: \hat{\theta}_{i}^{\mathrm{EB}} \pm t_{\mathrm{JY} 1} \sqrt{\widehat{\mathrm{MSE}}\left(\hat{\theta}_{i}^{\mathrm{EB}}\right)}
$$

where

$$
t_{\mathrm{JY} 1}=z_{\alpha / 2}+\left(z_{\alpha / 2}^{3}+z_{\alpha / 2}\right) \frac{\left(A+\psi_{i}\right) g_{3 \mathrm{JY} 1 i}}{8 A^{2}}
$$

and

$$
g_{3 J Y 1 i}(\hat{A})=g_{3 i}(A)-\frac{g_{2 i}(A)}{\left(A+\psi_{i}\right)^{2}} V(\hat{A}) .
$$

In this section, we compare six different CIs given in (3.4), (3.5), (3.6), (3.7), (3.8) and (3.9), which are referred as Method I, Method II, Method III, CIRao, CIJY and CIJY1, respectively. Method I and Method II represents the Cox (1975) and Prasad and Rao (1990) CIs for $\theta_{i}$. Both of them are naive methods and have a coverage accuracy $O\left(\mathrm{~m}^{-1}\right)$. However, Method III, CIRao, CIJY and CIJY1 are improved CIs with coverage accuracy $O\left(\mathrm{~m}^{-3 / 2}\right)$. The CPs and ALs of the proposed methods are comparable with Diao et al. (2013)'s CIs. However, CIRao and CIJY are constructed based on the proposed area specific MSEs. 
The simulation results in Tables 1-6 shows the estimated CPs and ALs of CIs for $\theta_{i}$ under normal and Laplace random effects distributions for number of small areas, $m=20$. For example, when $\hat{A}=\hat{A}_{P R}$ and pattern (III), Method I has a CP of $80.2 \%, 81.8 \%, 81.8 \%, 82.0 \%$ and $84.1 \%$ for G1, G2, G3, G4 and G5 respectively for normal random effects distribution. The CPs of the proposed methods are larger than Method I and Method II for all patterns. Method III, CIRao, CIJY and CIJY1 meet the nominal coverage rate more frequently than Method I and Method II. Thus, Method III, CIRao, CIJY and CIJY1 perform better than Method I and Method II in terms of coverage accuracy. However, the AL of these Methods is a bit larger than Method I and Method II for large sampling variances (G1 and G2). The proposed methods extend the widths of Method I and Method II so as to satisfy the nominal confidence level (Kubokawa ,2011).

We have also considered Laplace distribution to assess the robustness of the methods to possible deviations from the normality assumptions. In terms of CPs the proposed methods perform better than Method I and Method II. Method I has got a series under coverage problem (as low as 66.6\%, 66.4\%, 66.6\%, 66.6\% and $67.7 \%$ for G1, G2, G3, G4 and G5 respectively, values which are far below the $95 \%$ nominal value) for pattern (III) and when $A=\hat{A}_{P R}$ under Laplace distribution.

Figure 2 reports the CPs of CIs over a range of number of small areas $m$ for pattern (II). The CPs and ALs of CIs of all the methods are obtained through the above simulation exercise for areas such as $m=15,20,30,40,50,60,80$ and 100 . As the number of small areas $m$ increases the CPs increase and the ALs decrease for all methods and patterns. When $m \geq 60$, there is very little difference introduced by using any of the methods in terms of ALs and CPs for all patterns. All the methods except Method I perform better any time in terms of CPs and ALs. There is also no clear difference among the proposed methods in terms of CPs and ALs under the normal distribution. The percentage difference between CPs and ALs are negligible.

\subsection{CIs for the Difference of Two Small Area Means}

In this section we are interested in the difference of two small area means than in the specific values of the small area means themselves. The difference between estimated means (i.e., direct or EBLUP) gives information about the difference between population means. This is also an extension of Diao et al. (2013) CIs for $\theta_{i}-\theta_{j}$ where $i \neq j$ based on EBLUP estimator. In this paper, we develop a closed form area specific confidence intervals for $\theta_{i}-\theta_{j}$ based on $\hat{\theta}_{i}^{\mathrm{EB}}-\hat{\theta}_{j}^{\mathrm{EB}}$ under unequal sampling error variances and coverage probability is correct to $O\left(m^{-3 / 2}\right)$. We derive this theorem similar to Theorem 3.2. Note that the proof of this theorem can also be found in the Supplementary material. 
Theorem 3.2. For any $z>0$, the improved confidence interval is given by

$$
P\left[\theta_{i}-\theta_{j} \epsilon I^{\mathrm{CEBD}}(\hat{A})\right]=2 \Phi(z)-1-\frac{z \phi(z)}{4} \eta_{D}+O\left(m^{-3 / 2}\right),
$$

where

$$
\eta_{D}=\left(z^{2}+1\right)\left(\frac{A \psi_{i}}{A+\psi_{i}}+\frac{A \psi_{j}}{A+\psi_{j}}\right)^{-2}\left(\frac{\psi_{i}^{2}}{\left(A+\psi_{i}\right)^{2}}+\frac{\psi_{j}^{2}}{\left(A+\psi_{j}\right)^{2}}\right)^{2} V(\hat{A}) .
$$

Consider the following three corollaries that can be easily obtained from Theorem 3.2.

Corollary 3.4. The CP of $I^{\operatorname{CEBDRao}}(\hat{A})$ based on the Rao (2001) is written as

$$
P\left[\theta_{i}-\theta_{j} \in I^{\operatorname{CEBDRao}}(\hat{A})\right]=2 \Phi(z)-1-\frac{z \phi(z)}{4} \eta_{\text {RaoD }}+O\left(m^{-3 / 2}\right),
$$

where

$$
\eta_{\text {RaoD }}=\frac{\left(z_{\alpha / 2}^{2}+1\right)}{8}\left(g_{1 i}(A)+g_{1 j}(A)\right)^{-2} \frac{\left(\left(A+\psi_{i}\right) g_{3 \text { Rao } i}(A)+\left(A+\psi_{j}\right) g_{3 \operatorname{Rao} j}(A)\right)^{2}}{V(A)},
$$

$g_{3 \operatorname{Rao} i}(\hat{A})=\frac{\psi_{i}^{2}}{\left(\hat{A}+\psi_{i}\right)^{4}}\left(y_{i}-\mathbf{x}_{i}^{\prime} \hat{\boldsymbol{\beta}}\right)^{2} V(\hat{A})$ and $g_{3 \operatorname{Rao} j}(\hat{A})=\frac{\psi_{j}^{2}}{\left(\hat{A}+\psi_{j}\right)^{4}}\left(y_{j}-\mathbf{x}_{j}^{\prime} \hat{\boldsymbol{\beta}}\right)^{2} V(\hat{A})$.

Corollary 3.5. The CP of $I^{\mathrm{CEBDJY}}(\hat{A})$ based on JY is written as

$$
P\left[\theta_{i}-\theta_{j} \quad \in \quad I^{\mathrm{CEBDJY}}(\hat{A})\right]=2 \Phi(z)-1-\frac{z \phi(z)}{4} \eta_{\mathrm{JYD}}+O\left(m^{-3 / 2}\right),
$$

where

$$
\begin{gathered}
\eta_{\mathrm{JYD}}=\frac{\left(z_{\alpha / 2}^{2}+1\right)}{8}\left(g_{1 i}(A)+g_{1 j}(A)\right)^{-2} \frac{\left(\left(A+\psi_{i}\right) g_{3 \mathrm{JY} i}(A)+\left(A+\psi_{j}\right) g_{3 \mathrm{~J} Y j}(A)\right)^{2}}{V(A)} \\
g_{3 \mathrm{~J} Y i}(\hat{A})=\frac{\psi_{i}^{2}}{\left(\hat{A}+\psi_{i}\right)^{4}} \frac{\left(y_{i}-\mathbf{x}_{i}^{\prime} \tilde{\boldsymbol{\beta}}\right)^{2}}{\hat{A}+\psi_{i}-\mathbf{x}_{i}^{\prime}\left(\mathbf{X}^{\prime} \Sigma^{-1} \mathbf{X}\right)^{-1} \mathbf{x}_{i}} V(\hat{A})
\end{gathered}
$$

and

$$
g_{3 \mathrm{JY} j}(\hat{A})=\frac{\psi_{j}^{2}}{\left(\hat{A}+\psi_{j}\right)^{4}} \frac{\left(y_{j}-\mathbf{x}_{j}^{\prime} \hat{\beta}\right)^{2}}{\hat{A}+\psi_{j}-\mathbf{x}_{j}^{\prime}\left(\mathbf{X}^{\prime} \Sigma^{-1} \mathbf{X}\right)^{-1} \mathbf{x}_{j}} V(\hat{A}) .
$$


Corollary 3.6. The CP of $I^{\mathrm{CEBDJY1}}(\hat{A})$ based on JY1 is written as

$$
P\left[\theta_{i}-\theta_{j} \in I^{\mathrm{CEBDJY} 1}(\hat{A})\right]=2 \Phi(z)-1-\frac{z \phi(z)}{4} \eta_{\mathrm{JY} 1 \mathrm{D}}+O\left(m^{-3 / 2}\right),
$$

where

$$
\begin{gathered}
\eta_{\mathrm{JY} 1 \mathrm{D}}=\frac{\left(z_{\alpha / 2}^{2}+1\right)}{8}\left(g_{1 i}(A)+g_{1 j}(A)\right)^{-2} \frac{\left(\left(A+\psi_{i}\right) g_{3 \mathrm{JY} 1 i}(A)+\left(A+\psi_{j}\right) g_{33 \mathrm{Y} 1 j}(A)\right)^{2}}{V(A)} \\
g_{3 \mathrm{JY} 1 i}(A)=g_{3 i}(A)-g_{5 i}(A), \quad \text { where } \quad g_{5 i}(A)=\frac{g_{2 i}(A)}{\left(A+\psi_{i}\right)^{2}} V(\hat{A})
\end{gathered}
$$

and

$$
g_{3 \mathrm{JY} 1 j}(A)=g_{3 j}(A)-g_{5 j}(A), \quad \text { where } \quad g_{5 j}(A)=\frac{g_{2 j}(A)}{\left(A+\psi_{j}\right)^{2}} V(\hat{A}) .
$$

\subsubsection{Comparison of Confidence Intervals for the Difference between Two Small Area Means: Simulation Study}

The main purpose of this section is to find out which method performs best under normal and non normal random effects distributions. Here we have adopted the simulation set up of Section 3.1.1 with some modifications.

The two kinds of CIs for $\theta_{i}-\theta_{j}$ where $i \neq j$ are computed using the following formula:

Method ID (MetID) $: \hat{\theta}_{i}^{\mathrm{EB}}-\hat{\theta}_{j}^{\mathrm{EB}} \pm z_{\alpha / 2}\left(\psi_{i}^{1 / 2}\left(1-\hat{\gamma}_{i}\right)^{1 / 2}+\psi_{j}^{1 / 2}\left(1-\hat{\gamma}_{j}\right)^{1 / 2}\right)$,

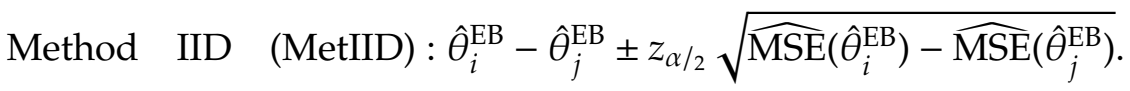

The improved CIs for $\theta_{i}-\theta_{j}$ where $i \neq j$ based on the EBLUP estimator in the FH model under unequal sampling variances consideration are given by

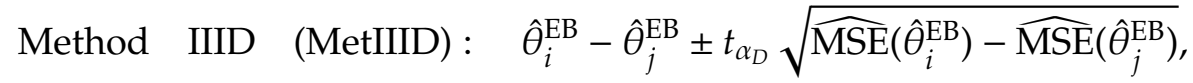

where $t_{\alpha_{D}}=z_{\alpha / 2}\left[\frac{\left(z_{\alpha / 2}^{2}+1\right)}{8}\left(g_{1 i}(A)+g_{1 j}(A)\right)^{-2} \frac{\left(\left(A+\psi_{i}\right) g_{3 i}(A)+\left(A+\psi_{j}\right) g_{3 j}(A)\right)^{2}}{V(A)}+1\right]$.

Similarly, the corresponding area specific versions of (3.12) are written as

$$
\mathrm{CIRaoD}: \hat{\theta}_{i}^{\mathrm{EB}}-\hat{\theta}_{j}^{\mathrm{EB}} \pm t_{\mathrm{RaoD}} \sqrt{\widehat{\operatorname{MSE}}\left(\hat{\theta}_{i}^{\mathrm{EB}}\right)-\widehat{\operatorname{MSE}}\left(\hat{\theta}_{j}^{\mathrm{EB}}\right)}
$$

where and

$$
\mathrm{CIJYD}: \hat{\theta}_{i}^{\mathrm{EB}}-\hat{\theta}_{j}^{\mathrm{EB}} \pm t_{\mathrm{JYD}} \sqrt{\widehat{\operatorname{MSE}}\left(\hat{\theta}_{i}^{\mathrm{EB}}\right)-\widehat{\operatorname{MSE}}\left(\hat{\theta}_{j}^{\mathrm{EB}}\right)}
$$


where $t_{\mathrm{JYD}}=z_{\alpha / 2}\left[\frac{\left(z_{\alpha / 2}^{2}+1\right)}{8}\left(g_{1 i}(A)+g_{1 j}(A)\right)^{-2} \frac{\left(\left(A+\psi_{i}\right) g_{3 \gamma Y i}(A)+\left(A+\psi_{j}\right) g_{33 Y j}(A)\right)^{2}}{V(A)}+1\right]$.

Another improved CIs for $\theta_{i}-\theta_{j}$ based on $\hat{\theta}_{i}^{\mathrm{EB}}-\hat{\theta}_{j}^{\mathrm{EB}}$ where $i \neq j$ is written as

$$
\text { CIJY1D : } \hat{\theta}_{i}^{\mathrm{EB}}-\hat{\theta}_{j}^{\mathrm{EB}} \pm t_{\mathrm{JY} 1 \mathrm{D}} \sqrt{\widehat{\mathrm{MSE}}\left(\hat{\theta}_{i}^{\mathrm{EB}}\right)-\widehat{\operatorname{MSE}}\left(\hat{\theta}_{j}^{\mathrm{EB}}\right)}
$$

where $t_{\mathrm{JY} 1 \mathrm{D}}=z_{\alpha / 2}\left[\frac{\left(z_{\alpha / 2}^{2}+1\right)}{8}\left(g_{1 i}(A)+g_{1 j}(A)\right)^{-2} \frac{\left(\left(A+\psi_{i}\right) g_{3 \zeta Y 1 i}(A)+\left(A+\psi_{j}\right) g_{3\} 11 j}(A)\right)^{2}}{V(A)}+1\right]$.

Similar to the previous case, in this section, we compare six different $C$ Is given in (3.10), (3.11), (3.12), (3.13), (3.14) and (3.15), which are referred as Method ID, Method IID, Method IIID, CIRaoD, CIJYD and CIJY1D, respectively. We see that the CPs of Method IIID, CIRaoD, CIJYD and CIJY1D behaved satisfactorily. They never differed from their $95 \%$ nominal value by more than $1.9 \%, 0.8 \%, 0.8 \%$ and $0.2 \%$ for FH, PR, ML and REML estimators, respectively, for pattern (II) and normal random effects distribution. The ALs of the proposed methods are a bit larger than Method ID and Method IID especially for large sampling variances (G1 and G2) and small sample sizes. This is to be expected since Method IIID, CIRaoD, CIJYD and CIJY1D increases the width of the intervals to meet the nominal coverage.

As far as the Laplace distribution is concerned, we see that for $m=20$ there is under coverage problem for all the methods, but the under coverage problem becomes more series for Method ID. For this distribution, the CPs of the proposed methods never differed from their $95 \%$ nominal value by more than $3.9 \%, 4.2 \%$, $3.2 \%$ and $3.5 \%$ (slightly below the $95 \%$ nominal value) for FH, PR, ML and REML estimators, respectively, for pattern (II). These findings are sensible for this heavy tail distribution. Like in the previous section, Figure 3 report CPs of the methods over a range of number of small areas, $m$, for pattern (II). As the number of small areas, $m$, increases the CPs increase for all the methods and patterns. The CPs and ALs for all variance patterns can be found in the Supplementary material.

\section{Application to Real Data}

In this section, we illustrate the proposed methods to estimate percentage of food expenditure by using the 2010/11 HCE and the 2007 census data sets of Ethiopia. Expenditure refers to total expenditure, including accommodation, food, purchases, travel, leisure activities and miscellaneous expenditure. The proposed CIs are used to estimate the percentage of food expenditure relative to total expenditure in 94 small areas (zones) in Ethiopia.

In 2012 , only $6.6 \%$ of consumer expenditure go to food consumed at home relative to total consumption expenditures in America. The amount of income 
spent on food fell from 17.5\% to 9.7\% between 1960 and 2007 in America (Morrison , 2014). Countries such as Spain, Norway, Belgium and France spend twice as much on food compared to America. Whereas countries like Mexico, China and Turkey spend three times as much on food compared to America. In contrast, people in poor countries are forced to spend the largest share of income on food (The Economist, 2013). The percentage of food expenditure spend on food in 2012 for some African countries such as Ethiopia, Kenya, Niger, Namibia and South Africa are 39.84\%, 47.44\%, 44.99\%, 24.36\% and $26.04 \%$, respectively ("Household consumption expenditure", 2014). Food prices started increasing in 2007 and exerting pressure on household budgets. Low income households spend a greater percentage of income on food (Carr et al. ,2014). If you spent very less on food, that leaves more money to things like health care, education, energy, home and saving.

\subsection{Generalized Variance Function}

We use the generalized variance function (GVF) to smooth out the uncertainty of the design based variance estimate. The factors $\exp \left(\frac{\hat{\sigma}^{2}}{2}\right)=1.29$ are the biascorrection terms in the log-linear analysis for the percentage of food expenditure estimates. Underestimation of the true variances will be occurred when we ignore the correction term in GVF method (Rivest and Belmonte , 2000; Rao , 2001). Figure 2 (top two) illustrates the GVF and variance in dots and stars for the percentage of food expenditure measures. From the figure we can easily observe that how the GVF smooth out the unreliable and noisy design based estimated variances (Esteban et al. ,2012; Hawala and Lahiri , 2010).

\subsection{Percentage of Food Expenditure Estimates}

The direct and model-based CI estimates are computed for the percentage of food expenditure measures. We present some of the CI estimates based on CIRao since almost all the proposed methods perform similarly. Zone3 50.03\% (95\% $\mathrm{CI}=47.21 \%-52.86 \%)$, Borena 51.10\% (95\% CI=48.26\% - 53.94\%), Itang 52.68\% (95\% CI $=49.88 \%-55.49 \%)$, Nuer $52.73 \%$ (95\% CI $=49.78 \%-55.68 \%)$ and Basketo $59.64 \%(95 \% \mathrm{CI}=56.74 \%-62.54 \%)$ spent the highest share of income on food (above 50\%). This result matches with other economical indicators, as they are quite deprived provinces where the percentage of food expenditure is important" Militino et al. (2012, page 2941). In contrast, zones such as Zone5 29.90\% (95\% $\mathrm{CI}=27.21 \%-32.61 \%$ ), Konso $30.71 \%$ (95\% CI=27.86\% - 33.56\%), Mekelle 32.54\% (95\% CI=29.73\% - 35.36\%), Nifas Silk 32.88\% (95\% CI=30.01\% - 35.74\%), Bahirdar $33.83 \%$ (95\% CI $=31.04 \%-36.61 \%)$, Akaki 34.26\% (95\% CI=31.43\% - 37.09\%) and 
Adama $35.14 \%$ (95\% CI=32.34\% - 37.94\%) spent the smallest share of income on food.

\subsection{Coverage Diagnostic}

This method evaluates the validity of the CIs based on EBLUP estimates. It is used to measure the overlap between the $95 \%$ CIs generated by the direct and the model based estimates in small area estimation procedure (Brown et al. ,2001). This test can be done as follows. Let $X_{1}$ and $X_{2}$ be two independent normal random variables having the same mean and variance $\sigma_{X_{1}}^{2}$ and $\sigma_{X_{2}}^{2}$ respectively. If $z(\alpha)$ is such that the probability that a standard normal variable takes values greater than $z(\alpha)$ is $\alpha / 2$, then a sufficient condition for there to be probability of $\alpha$ that the two intervals $X_{1} \pm z(\beta) \sigma_{X_{1}}$ and $X_{2} \pm z(\beta) \sigma_{X_{2}}$ do not overlap is when

$$
z(\beta)=z(\alpha)\left(1+\frac{\sigma_{X_{1}}}{\sigma_{X_{2}}}\right)^{-1} \sqrt{1+\frac{\sigma_{X_{1}}^{2}}{\sigma_{X_{2}}^{2}}}
$$

where $\sigma_{X_{1}}$ is the estimated standard error of the EBLUP estimate and $\sigma_{X_{2}}$ is the estimated standard error of the direct estimate, $z(\alpha)=1.96$. In our real data application $z(\beta) \approx 1.39$. This confirms that the overlap proportion is $95 \%$. In addition, Figure 1 shows that the CIs based on the EBLUP estimates lies within the CIs of the direct estimates.

\section{Conclusion}

The findings of this study indicate that there is no single method which dominates all the other methods in terms of the proposed methods. However, all the proposed methods have been numerically shown to be superior to the

Furthermore, CIs based on resampling approach do not have sensible empirical plug-in which is routinely used in place of the unknown parameters (Chatterjee and Lahiri , 2002). However, the advantages of our proposed methods are computational. Once we estimate the unknown parameters, then plug-in the estimates to MSEs and CIs.

The usefulness has also been shown through the application to the HCE survey data in Ethiopia to estimate percentage of food expenditure measures at the zone level. We derive zonal-level estimates of percentage of food expenditure measures by using small area estimation techniques to link data from the 2010/11 HCE and 2007 population and housing Census. 


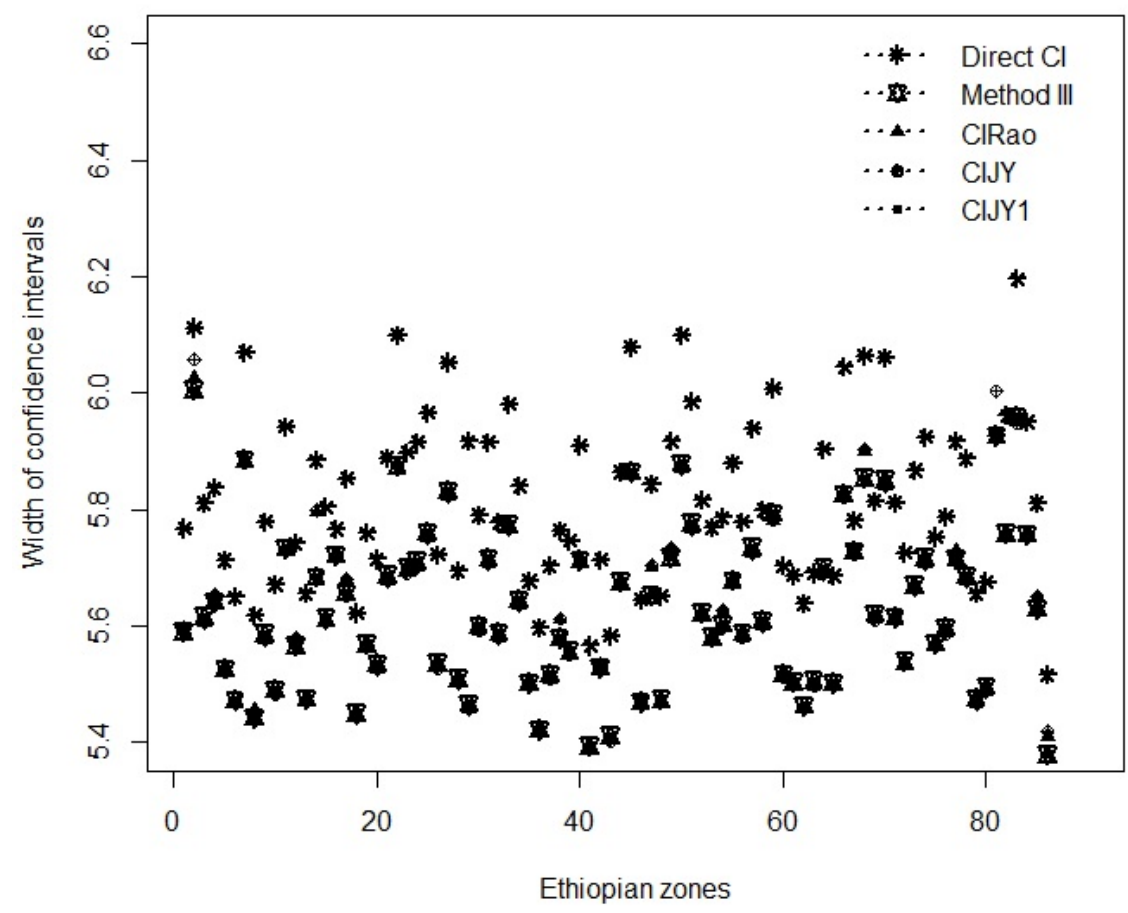

Figure 1: A plot illustrating the width of CIs of percentage of food expenditure estimates versus Ethiopian zones in 2010/11; Direct CI refers the CI based on the direct survey estimates. Method III, CIRao, CIJY and CIJY1 referring the CIs given by equations $(3.7-3.10)$.

\section{Acknowledgements}

The authors would like to thank the reviewers for their highly constructive and pinpoint comments which considerably improved the paper.

\section{References}

Ananya, R. (2007), Empirical and Hierarchical Bayesian Methods with Applications to Small Area Estimation. PhD Dissertation, University of Florida.

Brown, G., Chambers, R., Heady, P., Heasman, D. (2001), Evaluation of small area estimation methods - An application to unemployment estimates from 
the UK LFS, in Proceedings of Statistics Canada Symposium 2001: Achieving Data Quality in a Statistical Agency: A Methodological Perspective, Statistics Canada.

Carr, J., Lee, D., Scaife, A. and Hayes, I. (2014), Food statistics pocketbook 2013-in year update. Department of Environment, Food and Rural Affairs, UK.

Chatterjee, S. and Lahiri, P. (2002), Parametric bootstrap confidence interval in small area estimation problems. Unpublished report; University of Minnesota and University of Nebraska-Lincoln.

Chatterjee, S., Lahiri, P., and Li, H. (2008), Parametric bootstrap approximation to the distribution of EBLUP and related prediction intervals in linear mixed models. The Annals of Statistics, 36(3), 1221-1245.

Cox, D. R. (1975), Prediction intervals and empirical Bayes confidence intervals. In Perspectives in probability and statistics, Papers in honor of M.S. Bartlett (ed. J. Gani), pp. 47-55, Academic Press, London.

Datta, G.S. and Ghosh, M. (2012), Small area shrinkage estimation. Statistical Science, 27(1), 95-114.

Datta, G.S., Ghosh, M., Smith, D.D. and Lahiri, P. (2002), On an asymptotic theory of conditional and unconditional coverage probabilities of Empirical Bayes confidence intervals. Scandinavian Journal of Statistics, 29, 139-152.

Datta, G.S., Kubokawa, T., Molina, I. and Rao, J.N.K. (2011), Estimation of mean squared error of model-based small area estimators. Test, 20, 367-388.

Datta, G.S., and Lahiri, P. (2000), A unified measure of uncertainty of estimated best linear unbiased predictors in small area estimation problems. Statistica Sinica, 10, 613-627.

Datta, G.S., Rao, J.N.K. and Smith, D.D. (2005), On measuring the variability of small area estimators under a basic area level model. Biometrika, 92, 183-196.

Diao, L., Smith, D.D, Datta, G.S., Maiti, T. and Opsomer, J.D. (2013), Accurate confidence interval estimation of small area parameters under the Fay-Herriot model. Scandinavian Journal of Statistics, 41, 497-515.

Esteban, M.D., Morales, D., Perez, A. and Santamaria, L. (2012), Small area estimation of poverty proportions under area-level time models. Computational Statistics and Data Analysis, 56, 2840-2855.

Fay, R. E. and Herriot, R. A. (1979), Estimates of income for small places: An application of James-Stein procedure to census data. Journal of the American Statistical Association, 74, 269-277. 
Hall, P., Lee, S. M. and Young, G. A. (2000) Importance of interpolation when constructing double-bootstrap confidence intervals. Journal of the Royal Statistical Society, 62, 479-491.

Hall, P. and Maiti, T. (2006), On parametric bootstrap methods for small area prediction. Journal of the Royal Statistical Society B, 68, 221-238.

Hawala, S. and Lahiri, P. (2010), Variance modeling in the U.S. small area income and poverty estimates program for the American community survey. Section on Survey Methods-JSM, U.S. Census Bureau, United States.

Kubokawa, T. (2011), On Measuring Uncertainty of Small Area Estimation with Higher Order Accuracy, Journal of Japan Statistical Society, 41(2), 93-119.

Kubokawa, T. and Nagashima, B. (2012), Parametric bootstrap methods for bias correction in linear mixed models. Journal of Multivariate Analysis, 106, 1-16.

Li, H. (2006), An application of parametric bootstrap method in small area estimation problem. Proceedings of the American Statistical Association: Section on Survey Research Methods, University of Maryland, United States.

Militino, A.F., Goicoa, T. and Ugarte, M.D. (2012), Estimating the percentage of food expenditure in small areas using bias-corrected P-spline based estimators. Computational Statistics and Data Analysis, 56, 2934-2948.

Morrison, R.M. (2014), Selected charts 2014, Ag and food statistics: charting the essentials. Economic Research Service, USDA, Adminstrative Publication No. (Ag-067) 12pp.

Nankervis, J. C. (2005), Computational algorithms for double bootstrap confidence Intervals. Computational Statistics and Data Analysis, 49, 461-475.

Prasad, N.G.N. and Rao, J. N. K. (1990), The estimation of the mean squared error of small area estimators. Journal of the American Statistical Association, 85, 163-171.

Rao, J.N.K. (2001), EB and EBLUP in small area estimation in S. E. Ahmed and N. Reid (Eds.); Empirical Bayes and Likelihood Inference, Lecture Notes in Statistics 148, New York: Springer, 33-43.

Rao, J.N.K. (2003), Small Area Estimation. John Wiley and Sons, Inc., New York, 327 pages.

Rivest, L.P. and Belmonte, E. (2000), A Conditional Mean Squared Error of Small Area Estimators. Survey Methodology, 26, 79-90. 
Stein, C. (1981), Estimation of the mean of a multivariate normal distribution, Annals of Statistics, 9, 1135-1151.

\section{Appendices}

\section{Appendix A: Proof of Theorem 2.1}

Proof.

$$
\begin{aligned}
\tilde{g}_{3 J Y 1 i}(\hat{A})-\tilde{g}_{3 J Y 1 i}(A) & =\left(g_{3 i}(\hat{A})-g_{3 i}(A)\right)-\left(g_{5 i}(\hat{A})-g_{5 i}(A)\right) \\
& =I_{1}-I_{2} .
\end{aligned}
$$

According to Prasad and Rao (1990),

$$
E\left|I_{1}\right|=\left|g_{3 i}(\hat{A})-g_{3 i}(A)\right|=o\left(m^{-1}\right) .
$$

Let us show that $E\left|I_{2}\right|=o\left(m^{-1}\right)$.

$$
\begin{aligned}
g_{5 i}(A) & =\frac{g_{2 i}(A)}{\left(A+\psi_{i}\right)^{2}} V(\hat{A}) \\
& =\frac{\psi_{i}^{2}}{\left(A+\psi_{i}\right)^{4}} \mathbf{x}_{i}^{\prime}\left(\mathbf{X}^{\prime} \Sigma^{-1} \mathbf{X}\right)^{-1} \mathbf{x}_{i} V(\hat{A})
\end{aligned}
$$

For example, for the PR moment estimator, $V(\hat{A})=\frac{2}{m^{2}} \sum_{i=1}^{m}\left(A+\psi_{i}\right)^{2}$. Substituting reveals that

$$
\begin{gathered}
g_{5 i}(A)=\frac{2 \psi_{i}^{2} \sum_{i=1}^{m}\left(A+\psi_{i}\right)^{2}}{m^{2}\left(A+\psi_{i}\right)^{4}} \mathbf{x}_{i}^{\prime}\left(\mathbf{X}^{\prime} \Sigma^{-1} \mathbf{X}\right)^{-1} \mathbf{x}_{i} \\
I_{2}=g_{5 i}(\hat{A})-g_{5 i}(A) \\
=\frac{2 \psi_{i}^{2} \sum_{i=1}^{m}\left(\hat{A}+\psi_{i}\right)^{2}}{m^{2}\left(\hat{A}+\psi_{i}\right)^{4}} \mathbf{x}_{i}^{\prime}\left(\mathbf{X}^{\prime} \hat{\Sigma}^{-1} \mathbf{X}\right)^{-1} \mathbf{x}_{i}-\frac{2 \psi_{i}^{2} \sum_{i=1}^{m}\left(A+\psi_{i}\right)^{2}}{m^{2}\left(A+\psi_{i}\right)^{4}} \mathbf{x}_{i}^{\prime}\left(\mathbf{X}^{\prime} \Sigma^{-1} \mathbf{X}\right)^{-1} \mathbf{x}_{i} .
\end{gathered}
$$


Rewriting $I_{2}$

$$
\begin{aligned}
I_{2} & =\frac{2 \psi_{i}^{2} \sum_{i=1}^{m}\left(\hat{A}+\psi_{i}\right)^{2}}{m^{2}\left(\hat{A}+\psi_{i}\right)^{4}} \mathbf{x}_{i}^{\prime}\left(\mathbf{X}^{\prime} \hat{\boldsymbol{\Sigma}}^{-1} \mathbf{X}\right)^{-1} \mathbf{x}_{i}-\frac{2 \psi_{i}^{2} \sum_{i=1}^{m}\left(\hat{A}+\psi_{i}\right)^{2}}{m^{2}\left(\hat{A}+\psi_{i}\right)^{4}} \mathbf{x}_{i}^{\prime}\left(\mathbf{X}^{\prime} \boldsymbol{\Sigma}^{-1} \mathbf{X}\right)^{-1} \mathbf{x}_{i} \\
& +\frac{2 \psi_{i}^{2} \sum_{i=1}^{m}\left(\hat{A}+\psi_{i}\right)^{2}}{m^{2}\left(\hat{A}+\psi_{i}\right)^{4}} \mathbf{x}_{i}^{\prime}\left(\mathbf{X}^{\prime} \Sigma^{-1} \mathbf{X}\right)^{-1} \mathbf{x}_{i}-\frac{2 \psi_{i}^{2} \sum_{i=1}^{m}\left(A+\psi_{i}\right)^{2}}{m^{2}\left(A+\psi_{i}\right)^{4}} \mathbf{x}_{i}^{\prime}\left(\mathbf{X}^{\prime} \Sigma^{-1} \mathbf{X}\right)^{-1} \mathbf{x}_{i} \\
& =(2 / m) \psi_{i}^{2}\left(\hat{A}+\psi_{i}\right)^{-4}\left(\hat{A}^{2}+2 \hat{A} \bar{\psi}+\sum \psi_{i}^{2} / m\right)\left[\mathbf{x}_{i}^{\prime}\left(\mathbf{X}^{\prime} \hat{\boldsymbol{\Sigma}}^{-1} \mathbf{X}\right)^{-1} \mathbf{x}_{i}-\mathbf{x}_{i}^{\prime}\left(\mathbf{X}^{\prime} \boldsymbol{\Sigma}^{-1} \mathbf{X}\right)^{-1} \mathbf{x}_{i}\right] \\
& +\mathbf{x}_{i}^{\prime}\left(\mathbf{X}^{\prime} \boldsymbol{\Sigma}^{-1} \mathbf{X}\right)^{-1} \mathbf{x}_{i}\left[\left(2 / m^{2}\right) \psi_{i}^{2}\left(\hat{A}+\psi_{i}\right)^{-4} \sum_{i=1}^{m}\left(\hat{A}+\psi_{i}\right)^{2}\right. \\
& \left.-\left(2 / m^{2}\right) \psi_{i}^{2}\left(A+\psi_{i}\right)^{-4} \sum_{i=1}^{m}\left(A+\psi_{i}\right)^{2}\right] .
\end{aligned}
$$

Applying Taylor series expansion around A, we get

$$
\begin{aligned}
I_{3} & =\mathbf{x}_{i}^{\prime}\left(\mathbf{X}^{\prime} \hat{\Sigma}^{-1} \mathbf{X}\right)^{-1} \mathbf{x}_{i}-\mathbf{x}_{i}^{\prime}\left(\mathbf{X}^{\prime} \Sigma^{-1} \mathbf{X}\right)^{-1} \mathbf{x}_{i} \\
& =(\hat{A}-A)\left[\partial \mathbf{x}_{i}^{\prime}\left(\mathbf{X}^{\prime} \Sigma^{-1} \mathbf{X}\right)^{-1} \mathbf{x}_{i} / \partial A\right]_{A=A^{*}} \\
& =-(\hat{A}-A) \mathbf{x}_{i}^{\prime}\left(\mathbf{X}^{\prime} \Sigma^{*-1} \mathbf{X}\right)^{-1}\left(\mathbf{X}^{\prime} \Sigma^{*-2} \mathbf{X}\right)^{-1}\left(\mathbf{X}^{\prime} \Sigma^{*-1} \mathbf{X}\right)^{-1} \mathbf{x}_{i}
\end{aligned}
$$

and $E\left|I_{3}\right|=o\left(m^{-1}\right)$ (see Prasad and Rao, 1990).

It can also been shown that

$$
\begin{aligned}
\mathbf{x}_{i}^{\prime} & \left(\mathbf{X}^{\prime} \Sigma^{-1} \mathbf{X}\right)^{-1} \mathbf{x}_{i} \leq(1 / m)\left(A+\psi_{U}\right)\left(m \max _{i} \mathbf{x}_{i}^{\prime}\left(\mathbf{X}^{\prime} \Sigma^{-1} \mathbf{X}\right)^{-1} \mathbf{x}_{i}\right)=O\left(m^{-1}\right) \\
I_{4} & =\left(2 / m^{2}\right) \psi_{i}^{2}\left[\left(\hat{A}+\psi_{i}\right)^{-4} \sum_{i=1}^{m}\left(\hat{A}+\psi_{i}\right)^{2}-\left(A+\psi_{i}\right)^{-4} \sum_{i=1}^{m}\left(A+\psi_{i}\right)^{2}\right] \\
& =(2 / m) \psi_{i}^{2}\left(\hat{A}+\psi_{i}\right)^{-4}\left[(\hat{A}-A)^{2}+2(A+\bar{\psi})(\hat{A}-A)\right] \\
& +(2 / m) \psi_{i}^{2}\left(A^{2}+2 A \bar{\psi}+\sum \psi_{i}^{2} / m\right)\left[\left(\hat{A}+\psi_{i}\right)^{-4}-\left(A+\psi_{i}\right)^{-4}\right] \\
& =I_{5}+I_{6},
\end{aligned}
$$

where $\bar{\psi}=\sum_{i=1}^{m} \psi_{i} / m$.

Using a similar argument to Prasad and Rao (1990), it can be shown that

$$
E\left|I_{5}\right|=o\left(m^{-1}\right)
$$

and

$$
E\left|I_{6}\right|=o\left(m^{-1}\right) .
$$

Thus, $E\left|\tilde{g}_{3 J Y 1 i}(\hat{A})-\tilde{g}_{3 J Y 1 i}(A)\right|=o\left(m^{-1}\right)$. 


\section{Appendix B: Proof of Theorem 3.1}

Proof. The proof of the theorem about CIs based on the EBLUP estimator is given. The conditional distribution of $\theta_{i}$ given $y_{i}$ is given by

$$
\theta_{i} \mid y_{i} \sim N\left(\hat{\theta}_{i}^{\mathrm{EB}}, g_{1 i}(A)\right)
$$

where $\hat{\theta}_{i}^{\mathrm{EB}}=\left(1-\hat{\gamma}_{i}\right) y_{i}+\hat{\gamma}_{i} x_{i}^{\prime} \hat{\beta}, \quad \tilde{\theta}_{i}^{B}=\left(1-\gamma_{i}\right) y_{i}+\gamma_{i} x_{i}^{\prime} \tilde{\beta}, \quad g_{1 i}(A)=\frac{A \psi_{i}}{A+\psi_{i}}$.

Consider the following expression as

$$
P\left[\theta_{i} \leq \hat{\theta}_{i}^{\mathrm{EB}}+z \times \sqrt{\widehat{\mathrm{MSE}}\left(\hat{\theta}_{i}^{\mathrm{EB}}\right)}\right]=E[\Phi(z+F(z))]
$$

The symbols $\Phi(z)$ and $\phi(z)$ represents the distribution function and the probability density function, respectively, of the $N(0,1)$ distribution. Thus, the $\mathrm{CP}$ of $I^{\mathrm{CEB}}(\hat{A})$ is written as

$$
\begin{aligned}
P\left[\theta_{i} \in I^{\mathrm{CEB}}(\hat{A})\right] & =P\left[-z+F(-z)<\frac{\theta_{i}-\hat{\theta}_{i}^{\mathrm{EB}}}{\sqrt{g_{1 i}(A)}}<z+F(z)\right] \\
& =E[\Phi(z+F(z))-\Phi(-z+F(-z))]
\end{aligned}
$$

where $F(z)=\frac{z \times\left(\sqrt{\widehat{\mathrm{MSE}}\left(\hat{\theta}_{i}^{\mathrm{EB}}\right)}-\sqrt{g_{1 i}(A)}\right)+\hat{\theta}_{i}^{\mathrm{EB}}-\tilde{\theta}_{i}^{B}}{\sqrt{g_{1 i}(A)}}$.

Using the Taylor expansion with an integral remainder term, $\Phi(z+F(z))$ is evaluated as

$$
\begin{aligned}
\Phi(z+F(z)) & =\Phi(z)+F(z) \phi(z)+\frac{1}{2} F^{2}(z) \phi^{\prime}(z) \\
& +\frac{1}{2} \int_{z}^{z+F(z)}(z+F(z)-x)^{2} \phi^{\prime \prime}(x) d x \\
& =\Phi(z) \\
& +\left[F(z)-\frac{z}{2} F^{2}(z)\right] \phi(z)-\frac{1}{2} \int_{z}^{z+F(z)}(z+F(z)-x)^{2}\left(1-x^{2}\right) \phi(x) d x
\end{aligned}
$$

Taking expectation on both sides reveals that

$$
\begin{aligned}
E(\Phi(z+F(z))) & =\Phi(z)+E\left[F(z) \phi(z)-\frac{z}{2} F^{2}(z) \phi(z)\right. \\
& -\frac{1}{2} \int_{z}^{z+F(z)}(z+F(z)-x)^{2}\left(1-x^{2}\right) \phi(x) d x .
\end{aligned}
$$


First consider that

$$
\begin{aligned}
\hat{\theta}_{i}^{\mathrm{EB}}-\tilde{\theta}_{i}^{B} & =\left(1-\hat{\gamma}_{i}\right) y_{i}+\hat{\gamma}_{i} x_{i}^{\prime} \hat{\beta}-\left(1-\gamma_{i}\right) y_{i}-\gamma_{i} x_{i}^{\prime} \tilde{\beta} \\
& =\left(\gamma_{i}-\hat{\gamma}_{i}\right) r_{i}+\gamma_{i} x_{i}^{\prime}(\hat{\beta}-\tilde{\beta}),
\end{aligned}
$$

where $r_{i}=\left(y_{i}-x_{i}^{\prime} \hat{\beta}\right)$.Thus $F(z)$ can be decomposed into three components as

$$
\begin{aligned}
F(z) & =\frac{z \times\left(\sqrt{\widehat{\mathrm{MSE}}\left(\hat{\theta}_{i}^{\mathrm{EB}}\right)}-\sqrt{g_{1 i}(A)}\right)}{\sqrt{g_{1 i}(A)}}+\frac{\left(\gamma_{i}-\hat{\gamma}_{i}\right) r_{i}}{\sqrt{g_{1 i}(A)}}+\frac{\gamma_{i} x_{i}^{\prime}(\hat{\beta}-\tilde{\beta})}{\sqrt{g_{1 i}(A)}} \\
& =S_{1}+S_{2}+S_{3} \text { say. }
\end{aligned}
$$

Let us evaluate the expected value of each term as

$$
\begin{aligned}
E F(z) & =E\left(S_{1}+S_{2}+S_{3}\right) \\
& =E\left(S_{1}\right)+E\left(S_{2}\right)+E\left(S_{3}\right) .
\end{aligned}
$$

Applying the Taylor series expansion and taking expectation on both sides,

$$
\begin{aligned}
E S_{1} & =z \frac{1}{2}\left(g_{1 i}(A)\right)^{-1} E\left(\widehat{\operatorname{MSE}}\left(\hat{\theta}_{i}^{\mathrm{EB}}\right)-g_{1 i}(A)\right) \\
& -z \frac{1}{8}\left(g_{1 i}(A)\right)^{-2} E\left(\widehat{\operatorname{MSE}}\left(\hat{\theta}_{i}^{\mathrm{EB}}\right)-g_{1 i}(A)\right)^{2} \\
& +z \frac{3}{16}\left(g_{1 i}(A)\right)^{-1 / 2} E \int_{g_{1 i}(A)}^{\widehat{\mathrm{MSE}}\left(\hat{\theta}_{i}^{\mathrm{EB}}\right)} x^{-5 / 2}\left(\widehat{\operatorname{MSE}}\left(\hat{\theta}_{i}^{\mathrm{EB}}\right)-g_{1 i}(A)\right)^{2} d x .
\end{aligned}
$$

Let us compute the expectation of each term as

$$
\begin{aligned}
E\left(\widehat{\operatorname{MSE}}\left(\hat{\theta}_{i}^{\mathrm{EB}}\right)-g_{1 i}(A)\right) & =E\left(\widehat{\operatorname{MSE}}\left(\hat{\theta}_{i}^{\mathrm{EB}}\right)\right)-E\left(g_{1 i}(A)\right), \\
E\left(\widehat{\operatorname{MSE}}\left(\hat{\theta}_{i}^{\mathrm{EB}}\right)\right) & =\operatorname{MSE}\left(\hat{\theta}_{i}^{\mathrm{EB}}\right),
\end{aligned}
$$

since $E\left(\widehat{\operatorname{MSE}}\left(\hat{\theta}_{i}^{\mathrm{EB}}\right)\right)$ is nearly a second order unbiased estimator of $\operatorname{MSE}\left(\hat{\theta}_{i}^{\mathrm{EB}}\right)$. Thus, using the Taylor expansion, the second term can be expressed as

$$
\begin{gathered}
\widehat{\operatorname{MSE}}\left(\hat{\theta}_{i}^{\mathrm{EB}}\right)=\operatorname{MSE}\left(\hat{\theta}_{i}^{\mathrm{EB}}(A)\right)+\operatorname{MSE}\left(\hat{\theta}_{i}^{\mathrm{EB}}\left(A^{*}\right)\right)^{(1)}(\hat{A}-A)+O\left(m^{-3 / 2}\right), \\
E\left(\widehat{\operatorname{MSE}}\left(\hat{\theta}_{i}^{\mathrm{EB}}\right)-g_{1 i}(A)\right)^{2}=\left(g_{1 i}^{(1)}\left(A^{*}\right)\right)^{2} E(\hat{A}-A)^{2},
\end{gathered}
$$

where $g_{1 i}(A)=\frac{A \psi_{i}}{A+\psi_{i}}, g_{1 i}^{(1)}(A)=\frac{\psi_{i}}{A+\psi_{i}}-\frac{A \psi_{i}}{\left(A+\psi_{i}\right)^{2}}=\frac{\psi_{i}^{2}}{\left(A+\psi_{i}\right)^{2}},\left(g_{1 i}^{(1)}(A)\right)^{2}=\frac{\psi_{i}^{4}}{\left(A+\psi_{i}\right)^{4}}$. 
Substituting reveals that

$$
E\left(\widehat{\operatorname{MSE}}\left(\hat{\theta}_{i}^{\mathrm{EB}}\right)-g_{1 i}(A)\right)^{2}=\frac{\psi_{i}^{4}}{\left(A+\psi_{i}\right)^{4}} E(\hat{A}-A)^{2}+O\left(m^{-3 / 2}\right),
$$

where $E(\hat{A}-A)^{2}=V(\hat{A})+O_{p}\left(m^{-1}\right), V(\hat{A})$ can be the asymptotic variance of the $\mathrm{FH}, \mathrm{PR}, \mathrm{ML}$ and REML estimators of $A$. Substituting reveals that

$$
E\left(\widehat{\operatorname{MSE}}\left(\hat{\theta}_{i}^{\mathrm{EB}}\right)-g_{1 i}(A)\right)^{2}=\frac{\psi_{i}^{4}}{\left(A+\psi_{i}\right)^{4}} \frac{2}{m^{2}} \sum_{i=1}^{m}\left(A+\psi_{i}\right)^{2}+O\left(m^{-1}\right) .
$$

Let us compute the integral term using the same approach by Datta et al. (2002); Chatterjee and Lahiri (2002) and Diao et al. (2013) as follows.

$$
\begin{gathered}
E \int_{g_{1 i}(A)}^{\widehat{\operatorname{MSE}}\left(\hat{\theta}_{i}^{E B}\right)} x^{-5 / 2}\left(\widehat{\operatorname{MSE}}\left(\hat{\theta}_{i}^{E B}\right)-g_{1 i}(A)\right)^{2} d x=E I_{1}+E I_{2} \\
E I_{1}=\left[E \int_{g_{1 i}(A)}^{\widehat{\operatorname{MSE}}\left(\hat{\theta}_{i}^{E B}\right)} x^{-5 / 2}\left(\widehat{\operatorname{MSE}}\left(\hat{\theta}_{i}^{E B}\right)-g_{1 i}(A)\right)^{2} d x\right] I_{\left\{\widehat{\operatorname{MSE}}\left(\hat{\theta}_{i}^{E B}\right) \geq g_{1 i}(A)\right\}}
\end{gathered}
$$

and

$$
E I_{2}=\left[E \int_{g_{1 i}(A)}^{\widehat{\mathrm{MSE}}\left(\hat{\theta}_{i}^{E B}\right)} x^{-5 / 2}\left(\widehat{\operatorname{MSE}}\left(\hat{\theta}_{i}^{E B}\right)-g_{1 i}(A)\right)^{2} d x\right] I_{\left\{\widehat{\operatorname{MSE}}\left(\hat{\theta}_{i}^{E B}\right) \leq g_{1 i}(A)\right\}}
$$

But

$$
\begin{aligned}
E I_{1} & =\left[E \int_{g_{1 i}(A)}^{\widehat{\operatorname{MSE}}\left(\hat{\theta}_{i}^{E B}\right)} x^{-5 / 2}\left(\widehat{\operatorname{MSE}}\left(\hat{\theta}_{i}^{E B}\right)-g_{1 i}(A)\right)^{2} d x\right] I_{\left\{\widehat{\operatorname{MSE}}\left(\hat{\theta}_{i}^{E B}\right) \geq g_{1 i}(A)\right\}} \\
& \leq\left[E \int_{g_{1 i}(A)}^{\widehat{\operatorname{MSE}}\left(\hat{\theta}_{i}^{E B}\right)}\left(g_{1 i}(A)\right)^{-5 / 2}\left(\widehat{\operatorname{MSE}}\left(\hat{\theta}_{i}^{E B}\right)-g_{1 i}(A)\right)^{2} d x\right] I_{\left\{\widehat{\operatorname{MSE}}\left(\hat{\theta}_{i}^{E B}\right) \geq g_{1 i}(A)\right\}} \\
& \leq\left[\frac{1}{3}\left(g_{1 i}(A)\right)^{-5 / 2} E\left(\widehat{\operatorname{MSE}}\left(\hat{\theta}_{i}^{E B}\right)-g_{1 i}(A)\right)^{3}\right] \\
& =O\left(m^{\frac{-3}{2}}\right)
\end{aligned}
$$

since using Taylor series expansion $E\left(\widehat{\operatorname{MSE}}\left(\hat{\theta}_{i}^{E B}\right)-g_{1 i}(A)\right)^{3}=O\left(m^{\frac{-3}{2}}\right)$.

In order to evaluate $E I_{2}$, we choose $\epsilon_{m}=m^{-\alpha}(0<\alpha<1 / 2)$ (Datta et al. , 2002, see). According to Diao et al. (2013) we can rewrite $I_{\left\{\widehat{\operatorname{MSE}}\left(\hat{\theta}_{i}^{E B}\right) \leq g_{1 i}(A)\right\}}$ as

$$
I_{\left\{\widehat{\operatorname{MSE}}\left(\hat{\theta}_{i}^{E B}\right) \leq g_{1 i}(A)\right\}}=I_{\left\{\widehat{\operatorname{MSE}}\left(\hat{\theta}_{i}^{E B}\right) \leq g_{1 i}(A)-\epsilon_{m}\right\}}+I_{\left\{g_{1 i}(A)-\epsilon_{m} \leq \widehat{\operatorname{MSE}}\left(\hat{\theta}_{i}^{E B}\right) \leq g_{1 i}(A)\right\}}
$$


Then,

$$
\begin{aligned}
& {\left[E \int_{0}^{g_{1 i}(A)-\widehat{\operatorname{MSE}}\left(\hat{\theta}_{i}^{E B}\right)} x^{2}\left(\widehat{\operatorname{MSE}}\left(\hat{\theta}_{i}^{E B}\right)+x\right)^{-5 / 2} d x\right] I_{\left\{\widehat{\operatorname{MSE}}\left(\hat{\theta}_{i}^{E B}\right) \leq g_{1 i}(A)-\epsilon_{m}\right\}}} \\
& \leq\left[E \int_{0}^{g_{1 i}(A)-\widehat{\operatorname{MSE}}\left(\hat{\theta}_{i}^{E B}\right)} x^{4 / 2-5 / 2} d x\right] I_{\left\{\widehat{\operatorname{MSE}}\left(\hat{\theta}_{i}^{E B}\right) \leq g_{1 i}(A)-\epsilon_{m}\right\}} \\
& =E\left[2\left\{g_{1 i}(A)-\widehat{\operatorname{MSE}}\left(\hat{\theta}_{i}^{E B}\right)\right\}^{1 / 2} I_{\left\{\widehat{\operatorname{MSE}}\left(\hat{\theta}_{i}^{E B}\right) \leq g_{1 i}(A)-\epsilon_{m}\right\}}\right] \\
& \leq 2 \sqrt{E\left|g_{1 i}(A)-\widehat{\operatorname{MSE}}\left(\hat{\theta}_{i}^{E B}\right)\right|} \sqrt{P\left(g_{1 i}(A)-\widehat{\operatorname{MSE}}\left(\hat{\theta}_{i}^{E B}\right) \geq \epsilon_{m}\right)} \\
& \leq O\left(m^{-1 / 2}\right) E \sqrt{\frac{\left(g_{1 i}(A)-\widehat{\operatorname{MSE}}\left(\hat{\theta}_{i}^{E B}\right)\right)^{3}}{\epsilon_{m}^{3}}} \\
& =O\left(m^{\frac{-3}{2}}\right),
\end{aligned}
$$

since using Taylor series expansion $E\left(\widehat{\operatorname{MSE}}\left(\hat{\theta}_{i}^{E B}\right)-g_{1 i}(A)\right)^{3}=O\left(m^{\frac{-3}{2}}\right)$ and also

$$
\begin{aligned}
& E\left[\int_{\widehat{\operatorname{MSE}}\left(\hat{\theta}_{i}^{E B}\right)}^{g_{1 i}(A)} x^{-5 / 2}\left(x-\widehat{\operatorname{MSE}}\left(\hat{\theta}_{i}^{E B}\right)\right)^{2} d x I_{\left\{g_{1 i}(A)-\epsilon_{m} \leq \widehat{\operatorname{MSE}}\left(\hat{\theta}_{i}^{E B}\right) \leq g_{1 i}(A)\right\}}\right] \\
& \leq\left(g_{1 i}(A)-\epsilon_{m}\right)^{-5 / 2} E\left[\int_{\widehat{\operatorname{MSE}}\left(\hat{\theta}_{i}^{E B}\right)}^{g_{1 i}(A)}\left(x-\widehat{\operatorname{MSE}}\left(\hat{\theta}_{i}^{E B}\right)\right)^{2} d x I_{\left\{g_{1 i}(A)-\epsilon_{m} \leq \widehat{\operatorname{MSE}}\left(\hat{\theta}_{i}^{E B}\right) \leq g_{1 i}(A)\right\}}\right] \\
& \quad \leq \frac{1}{3}\left(g_{1 i}(A)-\epsilon_{m}\right)^{-5 / 2} E\left(g_{1 i}(A)-\widehat{\operatorname{MSE}}\left(\hat{\theta}_{i}^{E B}\right)\right)^{3} \\
& =O\left(m^{\frac{-3}{2}}\right)
\end{aligned}
$$

since using Taylor series expansion $E\left(\widehat{\operatorname{MSE}}\left(\hat{\theta}_{i}^{E B}\right)-g_{1 i}(A)\right)^{3}=O\left(m^{\frac{-3}{2}}\right)$.Thus, $E I_{1}+$ $E I_{2}=O\left(m^{\frac{-3}{2}}\right)$.

Let us compute $S_{2}$ as

$$
S_{2}=\frac{\left(\gamma_{i}-\hat{\gamma}_{i}\right)\left(y_{i}-x_{i}^{\prime} \hat{\beta}\right)}{\sqrt{g_{1 i}(A)}} .
$$

By the Taylor expansion, $\gamma_{i}(\hat{A})=\gamma_{i}(A)+\gamma_{i}^{(1)}\left(A^{*}\right)(\hat{A}-A)$ and $\hat{\beta}=\tilde{\beta}(A)+\tilde{\beta}^{(1)}\left(A^{*}\right)(\hat{A}-$ $A)$, where $\left|A^{*}-A\right| \leq|\hat{A}-A|$ and $\gamma_{i}^{(1)}\left(A^{*}\right)$ is the derivative of $\tilde{\beta}(A)$ evaluated at $A=A^{*}$, similarly $\tilde{\beta}^{(1)}\left(A^{*}\right)$ is the derivative of $\gamma_{i}(A)$ evaluated at $A=A^{*}$. Thus, $\gamma_{i}(\hat{A})-\gamma_{i}(A)=\gamma_{i}^{(1)}(\hat{A}-A)$ and $\hat{\beta}-\tilde{\beta}(A)=\tilde{\beta}^{(1)}(\hat{A}-A)$. Substituting reveals that

$$
\left.S_{2}=-g_{1 i}^{-1 / 2} \gamma_{i}^{(1)}\left((\hat{A}-A)\left(y_{i}-x_{i}^{\prime} \tilde{\beta}(A)\right)-(\hat{A}-A) x_{i}^{\prime} \tilde{\beta}^{(1)}(\hat{A}-A)\right)\right) .
$$


Taking expectation on both sides,

$$
\begin{aligned}
E S_{2} & =-g_{1 i}^{-1 / 2} E\left[\left(\hat{\gamma}_{i}-\gamma_{i}\right)\left(y_{i}-x_{i}^{\prime} \hat{\beta}\right)\right] \\
& =-g_{1 i}^{-1 / 2} \gamma_{i}^{(1)} E\left[(\hat{A}-A)\left(y_{i}-x_{i}^{\prime} \tilde{\beta}(A)\right)\right]+g_{1 i}^{-1 / 2} \gamma_{i}^{(1)} E\left[(\hat{A}-A) x_{i}^{\prime} \tilde{\beta}^{(1)}(\hat{A}-A)\right] .
\end{aligned}
$$

Let the first part can be written as

$$
\begin{aligned}
S_{2}^{*} & =-g_{1 i}^{-1 / 2} \gamma_{i}^{(1)} E\left[(\hat{A}-A)\left(y_{i}-x_{i}^{\prime} \tilde{\beta}(A)\right)\right] \\
& =-g_{1 i}^{-1 / 2} \gamma_{i}^{(1)} E\left[(\hat{A}-A)\left(y_{i}-x_{i}^{\prime} \beta\right)\right]+o\left(m^{-1}\right) .
\end{aligned}
$$

Using Stein identity (see equation (8)), $E S_{2}^{*}=-g_{1 i}^{-1 / 2} \gamma_{i}^{(1)}\left(A+\psi_{i}\right) E\left(\frac{\partial \hat{A}}{\partial y_{i}}\right)=0$, since $\frac{\partial \hat{A}}{\partial y_{i}}=O_{P}\left(m^{-1}\right)$. The expected value of $S_{3}$ can be evaluated as

$$
S_{3}=g_{1 i}(A)^{-1 / 2} \gamma_{i} x_{i}^{\prime}\left(\sum_{i=1}^{m} \frac{x_{i} x_{i}^{\prime}}{A+\psi_{i}}\right)^{-1} \sum_{i=1}^{m} \frac{x_{i}}{A+\psi_{i}} e_{i},
$$

since $\tilde{\beta}=\left(\sum_{i=1}^{m} \frac{x_{i} x_{i}^{\prime}}{A+\psi_{i}}\right)^{-1} \sum_{i=1}^{m} \frac{x_{i}}{A+\psi_{i}} y_{i}$,

$$
E\left(S_{3}\right)=0 .
$$

Combination of the above results, we have

$$
\begin{aligned}
& E F(z)= E\left(S_{1}\right)+E\left(S_{2}\right)+E\left(S_{3}\right) \\
&= E\left(S_{1}\right)+0+0 \\
&= \frac{z}{2}\left(g_{1 i}(A)\right)^{-1}\left(g_{2 i}(A)+g_{3 i}(A)\right)-\frac{z}{8}\left(g_{1 i}(A)\right)^{-2} \frac{\psi_{i}^{4}}{\left(A+\psi_{i}\right)^{4}} V(\hat{A})+O\left(m^{-3 / 2}\right) \\
&= \frac{z}{2}\left(\frac{A \psi_{i}}{A+\psi_{i}}\right)^{-1}\left(\frac{\psi_{i}^{2}}{\left(A+\psi_{i}\right)^{2}} x_{i}^{\prime}\left(\sum_{i=1}^{m} \frac{x_{i} x_{i}^{\prime}}{A+\psi_{i}}\right)^{-1} x_{i}+\frac{\psi_{i}^{2}}{\left(A+\psi_{i}\right)^{3}} V(\hat{A})\right) \\
&- \frac{z}{8}\left(\frac{A \psi_{i}}{A+\psi_{i}}\right)^{-2} \frac{\psi_{i}^{4}}{\left(A+\psi_{i}\right)^{4}} V(\hat{A})+O\left(m^{-3 / 2}\right) . \\
& E F(z)^{2}=E\left(S_{1}+S_{2}+S_{3}\right)^{2} \\
& \quad \quad=E\left(S_{1}^{2}\right)+E\left(S_{2}^{2}\right)+E\left(S_{3}^{2}\right)+2 E\left(S_{1} S_{2}\right)+2 E\left(S_{1} S_{3}\right)+2 E\left(S_{2} S_{3}\right) .
\end{aligned}
$$

We compute all the expectation values turn by turn as follows.

Let us evaluate $S_{1} S_{2}$ using the Taylor expansion as

$$
\begin{aligned}
S_{1} S_{2} & =\frac{z \times\left(\sqrt{\widehat{\mathrm{MSE}}\left(\hat{\theta}_{i}^{\mathrm{EB}}\right)}-\sqrt{g_{1 i}(A)}\right)\left(\gamma_{i}-\hat{\gamma}_{i}\right)\left(y_{i}-x_{i}^{\prime} \hat{\beta}\right)}{g_{1 i}(A)} \\
& =g_{1 i}^{-1}(A) \frac{-z}{2}\left(\widehat{\operatorname{MSE}}\left(\hat{\theta}_{i}^{\mathrm{EB}}\right)-g_{1 i}(A)\right)\left(\gamma_{i}-\hat{\gamma}_{i}\right)\left(y_{i}-x_{i}^{\prime} \beta\right) \\
& =-g_{1 i}^{-1}(A) \frac{z}{2} g_{1 i}^{(1)}(A) \gamma_{i}^{(1)}(A)\left((\hat{A}-A)^{2}\left(y_{i}-x_{i}^{\prime} \tilde{\beta}(A)\right)-(\hat{A}-A)^{3} x_{i}^{\prime} \tilde{\beta}^{(1)}(A)\right) .
\end{aligned}
$$


Letting $c=-g_{1 i}^{-1}(A) \frac{z}{2} g_{1 i}^{(1)}(A) \gamma_{i}^{(1)}(A)$ and taking expectation on both sides gives that

$$
E S_{1} S_{2}=c E\left((\hat{A}-A)^{2}\left(y_{i}-x_{i}^{\prime} \tilde{\beta}(A)\right)\right)-c E\left((\hat{A}-A)^{3} x_{i}^{\prime} \tilde{\beta}^{(1)}(A)\right)=r_{11}+r_{12},
$$

say,

$$
\begin{aligned}
E r_{11} & =c E\left((\hat{A}-A)^{2}\left(y_{i}-x_{i}^{\prime} \tilde{\beta}(A)\right)\right) \\
& =c E\left((\hat{A}-A)^{2}\left(y_{i}-x_{i}^{\prime} \beta\right)\right)+o\left(m^{-1}\right) .
\end{aligned}
$$

Using Stein identity (see equation (8)),

$$
\begin{aligned}
E r_{11} & =c\left(A+\psi_{i}\right) E\left(\frac{\partial}{\partial y_{i}}(\hat{A}-A)^{2}\right) \\
& =c\left(A+\psi_{i}\right) E\left(2(\hat{A}-A) \frac{\partial \hat{A}}{\partial y_{i}}\right),
\end{aligned}
$$

but $\frac{\partial \hat{A}}{\partial y_{i}}=O_{P}\left(m^{-1}\right)$.

$$
\begin{aligned}
E r_{12} & =c E\left((\hat{A}-A)^{3} x_{i}^{\prime} \tilde{\beta}^{(1)}(A)\right) \\
& =c x_{i}^{\prime} \tilde{\beta}^{(1)}(A) E(\hat{A}-A)^{3} .
\end{aligned}
$$

Since $E(\hat{A}-A)^{2 S}=O\left(m^{-S}\right)$, (Prasad and Rao, 1990), thus $E(\hat{A}-A)^{3}=O\left(m^{-3 / 2}\right)$.

Let us evaluate $S_{2} S_{3}$ as using the Taylor series expansion as

$$
\begin{aligned}
S_{2} S_{3} & =g_{1 i}^{-1}\left(y_{i}-x_{i}^{\prime} \hat{\beta}\right) \gamma_{i} x_{i}^{\prime}(\hat{\beta}-\tilde{\beta})\left(\gamma_{i}-\hat{\gamma}_{i}\right) \\
& =-g_{1 i}^{-1}\left(y_{i}-x_{i}^{\prime} \tilde{\beta}(A)\right) \gamma_{i} x_{i}^{\prime} \tilde{\beta}^{(1)}(A)(\hat{A}-A) \gamma_{i}^{(1)}(A)(\hat{A}-A) \\
& +g_{1 i}^{-1} x_{i}^{\prime} \tilde{\beta}^{(1)}(A)(\hat{A}-A) \gamma_{i} x_{i}^{\prime} \tilde{\beta}^{(1)}(A)(\hat{A}-A) \gamma_{i}^{(1)}(A)(\hat{A}-A) \\
& =r_{21}+r_{22} .
\end{aligned}
$$

Taking expectation on both sides gives

$$
\begin{aligned}
E r_{21} & =-g_{1 i}^{-1} \gamma_{i}^{(1)}(A) \gamma_{i} E\left(\left(y_{i}-x_{i}^{\prime} \tilde{\beta}(A)\right) x_{i}^{\prime} \tilde{\beta}^{(1)}(A)(\hat{A}-A)^{2}\right) \\
& =-g_{1 i}^{-1} \gamma_{i}^{(1)}(A) \gamma_{i} E\left(\left(y_{i}-x_{i}^{\prime} \beta\right) x_{i}^{\prime} \tilde{\beta}^{(1)}(A)(\hat{A}-A)^{2}\right)+o\left(m^{-1}\right) .
\end{aligned}
$$

Using Stein identity (see equation (8)),

$$
E r_{21}=-g_{1 i}^{-1} \gamma_{i}^{(1)}(A) \gamma_{i}\left(A+\psi_{i}\right) E\left(\frac{\partial}{\partial y_{i}}\left\{x_{i}^{\prime} \tilde{\beta}^{(1)}(A)(\hat{A}-A)^{2}\right\}\right)+o\left(m^{-1}\right)
$$


Note that

$$
\begin{aligned}
& \frac{\partial}{\partial y_{i}}\left\{x_{i}^{\prime} \tilde{\beta}^{(1)}(A)(\hat{A}-A)^{2}\right\} \\
& =\frac{\partial}{\partial y_{i}}\left\{x_{i}^{\prime} \tilde{\beta}^{(1)}(A)\right\}(\hat{A}-A)^{2}+2 x_{i}^{\prime} \tilde{\beta}^{(1)}(A)(\hat{A}-A) \frac{\partial \hat{A}}{\partial y_{i}},
\end{aligned}
$$

where

$$
\begin{gathered}
\frac{\partial}{\partial y_{i}}\left\{x_{i}^{\prime} \tilde{\beta}^{(1)}(A)\right\}=\frac{\partial}{\partial A} x_{i}^{\prime}\left\{\frac{\partial}{\partial y_{i}} \tilde{\beta}(A)\right\} \\
=\frac{\partial}{\partial A}\left\{\frac{\tilde{h}_{i i}}{A+\psi_{i}}\right\}+O\left(m^{-1}\right)
\end{gathered}
$$

in which $\tilde{h}_{i i}=x_{i}^{\prime}\left\{\sum_{j=1}^{m}\left(A+\psi_{j}\right)^{-1} x_{j} x_{j}^{\prime}\right\}^{-1}$ is $O\left(m^{-1}\right), \frac{\partial \hat{A}}{\partial y_{i}}=O_{P}\left(m^{-1}\right)$ and $\frac{\partial}{\partial y_{i}}(\hat{\beta}(\hat{A}))=$ $O_{P}\left(m^{-1}\right)$ (see Kubokawa (2011, page 14) and Datta et al. (2011, page 20)).

$$
\begin{aligned}
E r_{22} & =g_{1 i}^{-1} x_{i}^{\prime} \tilde{\beta}^{(1)}(A)(\hat{A}-A) \gamma_{i} x_{i}^{\prime} \tilde{\beta}^{(1)}(A)(\hat{A}-A) \gamma_{i}^{(1)}(A)(\hat{A}-A) \\
& =g_{1 i}^{-1} \gamma_{i} \gamma_{i}^{(1)}(A)\left\{x_{i}^{\prime} \tilde{\beta}^{(1)}(A)\right\}^{2} E(\hat{A}-A)^{3}=O\left(m^{-3 / 2}\right) .
\end{aligned}
$$

Thus, $E\left(S_{2} S_{3}\right)=O\left(m^{-3 / 2}\right)$.

Let us evaluate $E\left(S_{1} S_{3}\right)$ as

$$
S_{1} S_{3}=\frac{z \times\left(\sqrt{\widehat{\operatorname{MSE}}\left(\hat{\theta}_{i}^{\mathrm{EB}}\right)}-\sqrt{g_{1 i}(A)}\right) \gamma_{i} x_{i}^{\prime}(\hat{\beta}-\tilde{\beta})}{g_{1 i}(A)} .
$$

Using the Taylor expansion we have $=g_{1 i}^{-1} z \gamma_{i} g_{1 i}^{(1)} x_{i}^{\prime} \tilde{\beta}^{(1)}(\hat{A}-A)^{2}$. Then,

$$
\begin{aligned}
E\left(S_{1} S_{3}\right) & =g_{1 i}^{-1} z \gamma_{i} g_{1 i}^{(1)} x_{i}^{\prime} \tilde{\beta}^{(1)} E(\hat{A}-A)^{2} \\
& =g_{1 i}^{-1} z \gamma_{i} g_{1 i}^{(1)} x_{i}^{\prime} \tilde{\beta}^{(1)} \operatorname{Var}(\hat{A}) .
\end{aligned}
$$

We know that

$$
\begin{aligned}
S_{1}^{2} & =\left(\frac{z\left(\sqrt{\widehat{\operatorname{MSE}}\left(\hat{\theta}_{i}^{\mathrm{EB}}\right)}-\sqrt{g_{1 i}(A)}\right)}{\sqrt{g_{1 i}(A)}}\right)^{2} \\
& =\frac{z^{2}}{4}\left(g_{1 i}^{-1}(A)\left(\widehat{\mathrm{MSE}}\left(\hat{\theta}_{i}^{\mathrm{EB}}\right)-g_{1 i}(A)\right)\right)^{2} .
\end{aligned}
$$


Using the Taylor expansion, we have

$$
S_{1}^{2}=\frac{z^{2}}{4}\left(g_{1 i}^{-1}(A)\left(\widehat{\operatorname{MSE}}\left(\hat{\theta}_{i}^{\mathrm{EB}}\right)-g_{1 i}(A)\right)\right)^{2} .
$$

Taking expectation on both sides,

$$
\begin{aligned}
E S_{1}^{2} & =\frac{z^{2}}{4} E\left(g_{1 i}^{-1}(A)\left(g_{1 i}^{1}(A)(\hat{A}-A)\right)\right)^{2}+O\left(m^{-3 / 2}\right) \\
& =\frac{z^{2}}{4} g_{1 i}^{-2}(A)\left(g_{1 i}^{1}(A)\right)^{2} E(\hat{A}-A)^{2}+O\left(m^{-3 / 2}\right) \\
& =\frac{z^{2} \psi_{i}^{2}}{4 A^{2}\left(A+\psi_{i}\right)^{2}} V(\hat{A})+O\left(m^{-3 / 2}\right) .
\end{aligned}
$$

Let us evaluate $S_{2}^{2}=g_{1 i}^{-1}\left(\gamma_{i}-\hat{\gamma}_{i}\right)^{2}\left(y_{i}-x_{i}^{\prime} \hat{\beta}\right)^{2}$. By the Taylor expansion,

$$
\begin{aligned}
S_{2}^{2} & =g_{1 i}^{-1}\left(\gamma_{i}-\hat{\gamma}_{i}\right)^{2}\left(y_{i}-x_{i}^{\prime} \hat{\beta}\right)^{2} \\
& =g_{1 i}^{-1}\left(\gamma_{i}^{(1)}(A)\right)^{2}\left(y_{i}-x_{i}^{\prime} \tilde{\beta}(A)\right)^{2}(\hat{A}-A)^{2} \\
& -2 g_{1 i}^{-1}\left(\gamma_{i}^{(1)}(A)\right)^{2} x_{i}^{\prime} \tilde{\beta}^{(1)}(A)\left(y_{i}-x_{i}^{\prime} \tilde{\beta}(A)\right)(\hat{A}-A)^{3} \\
& +\left(x_{i}^{\prime} \tilde{\beta}^{(1)}(A)\right)^{2}(\hat{A}-A)^{4} \\
& =r_{31}+r_{32}+r_{33} .
\end{aligned}
$$

Taking expectation on both sides,

$$
\begin{aligned}
E r_{31} & =g_{1 i}^{-1}\left(\gamma_{i}^{(1)}(A)\right)^{2} E\left\{\left(y_{i}-x_{i}^{\prime} \tilde{\beta}(A)\right)^{2}(\hat{A}-A)^{2}\right\} \\
& =g_{1 i}^{-1}\left(\gamma_{i}^{(1)}(A)\right)^{2} E\left\{\left(y_{i}-x_{i}^{\prime} \beta\right)^{2}(\hat{A}-A)^{2}\right\}+o\left(m^{-1}\right) .
\end{aligned}
$$

Using Stein identity (see equation (8)),

$$
\begin{aligned}
& E\left[(\hat{A}-A)^{2}\left(y_{i}-x_{i}^{\prime} \beta\right)^{2}\right]=\left(A+\psi_{i}\right) E\left[\frac{\partial}{\partial y_{i}}\left\{(\hat{A}-A)^{2}\left(y_{i}-x_{i}^{\prime} \beta\right)\right\}\right] \\
&=\left(A+\psi_{i}\right) \\
& \times E\left[2(\hat{A}-A)\left(y_{i}-x_{i}^{\prime} \beta\right) \frac{\partial \hat{A}}{\partial y_{i}}+(\hat{A}-A)^{2} \frac{\partial}{\partial y_{i}}\left\{\left(y_{i}-x_{i}^{\prime} \beta\right)\right\}\right] \\
&+o\left(m^{-1}\right) \\
&=\left(A+\psi_{i}\right) E(\hat{A}-A)^{2}+o\left(m^{-1}\right), \\
& E r_{31}= g_{1 i}^{-1}\left(\gamma_{i}^{(1)}(A)\right)^{2}\left(A+\psi_{i}\right) E(\hat{A}-A)^{2}+o\left(m^{-1}\right), \\
& E r_{32}=2 g_{1 i}^{-1}\left(\gamma_{i}^{(1)}(A)\right)^{2} x_{i}^{\prime} \tilde{\beta}^{(1)}(A) E\left\{\left(y_{i}-x_{i}^{\prime} \beta\right)(\hat{A}-A)^{3}\right\}+o\left(m^{-1}\right) \\
&=6 g_{1 i}^{-1}\left(\gamma_{i}^{(1)}(A)\right)^{2} x_{i}^{\prime} \tilde{\beta}^{(1)}(A)\left(A+\psi_{i}\right) E(\hat{A}-A)^{2} \frac{\partial \hat{A}}{\partial y_{i}}+o\left(m^{-1}\right), \\
& E_{33}=\left(x_{i}^{\prime} \tilde{\beta}^{(1)}(A)\right)^{2} E(\hat{A}-A)^{4}=O\left(m^{-2}\right),
\end{aligned}
$$


since $E(\hat{A}-A)^{2 S}=O\left(m^{-S}\right)$. Substituting reveals that

$$
\begin{aligned}
E S_{2}^{2} & =E r_{31}+E r_{32}+E r_{33} \\
& =g_{1 i}^{-1}\left(\gamma_{i}^{(1)}\right)^{2}\left(A+\psi_{i}\right) E(\hat{A}-A)^{2}+O\left(m^{-2}\right) \\
& =\frac{\psi_{i}}{A\left(A+\psi_{i}\right)^{2}} V(\hat{A})+O\left(m^{-2}\right)
\end{aligned}
$$

since $\left(\gamma_{i}^{(1)}\right)^{2}=\frac{\psi_{i}^{2}}{\left(A+\psi_{i}\right)^{4}}$ and $E(\hat{A}-A)^{2}=V(\hat{A})+o_{p}\left(m^{-1}\right)$. Using this fact, we obtain

$$
E S_{2}^{2}=\frac{\psi_{i}}{A\left(A+\psi_{i}\right)^{2}} V(\hat{A})+O\left(m^{-2}\right)
$$

Similarly, we have

$$
\begin{aligned}
& E\left(S_{3}^{2}\right)=\frac{\psi_{i}}{A\left(A+\psi_{i}\right)} x_{i}^{\prime}\left(\sum_{j=1}^{m}\left(A+\psi_{j}\right)^{-1} x_{j} x_{j}^{\prime}\right)^{-1} x_{i} \\
& E\left(S_{3}^{3}\right)=O\left(m^{-2}\right),
\end{aligned}
$$

where $\operatorname{Var}\left(e_{i}\right)=\psi_{i}$. The remainder term $\int_{z}^{z+F(z)}(z+F(z)-x)^{2}\left(x^{2}-1\right) \phi(x) d x$ can be simplified using the approach by Datta et al. (2002), Chatterjee and Lahiri (2002) and Diao et al. (2013) as follows. Since within the limits of the integral $0<|z+F(z)-x|<|F(z)|$ and $\left|\left(x^{2}-1\right) \phi(x)\right|<2 \phi(\sqrt{3})$, it follows that

$$
\begin{aligned}
& E\left|\int_{z}^{z+F(z)}\left(x^{2}-1\right)(z+F(z)-x)^{2} \phi(x) d x\right| \\
& \quad \leq E|F(z)|^{2}\left|\int_{z}^{z+F(z)} 2 \phi(\sqrt{3}) d x\right| \\
& \quad=2 E|F(z)|^{3} \phi(\sqrt{3}) \\
& =O\left(m^{-3 / 2}\right) .
\end{aligned}
$$

Finally, combination and simplification of all the above expressions give the $\mathrm{CP}$ of $I^{\mathrm{CEB}}(\hat{A})$ as

$$
P\left[\theta_{i} \in I^{\mathrm{CEB}}(\hat{A})\right]=2 \Phi(z)-1-\frac{z \phi(z)}{4} \eta+O\left(m^{-3 / 2}\right),
$$

where

$$
\eta=\left(z^{2}+1\right) \frac{\psi_{i}^{2}}{A^{2}\left(A+\psi_{i}\right)^{2}} V(\hat{A}) .
$$




\section{Appendix C: Proof of Theorem 3.2}

Proof. The $\mathrm{CP}$ of $I^{\mathrm{CEB}}(\hat{A})$ is written as

$$
\begin{aligned}
P\left[\theta_{i}-\theta_{j} \in I^{\mathrm{CEB}}(\hat{A})\right] & =P\left[-z+F(-z)<\frac{\theta_{i}-\theta_{j}-\left(\hat{\theta}_{i}^{\mathrm{EB}}-\hat{\theta}_{j}^{\mathrm{EB}}\right)}{\sqrt{g_{1 i}(A)+g_{2 j}(A}}<z+F(z)\right] \\
& =E[\Phi(z+F(z))-\Phi(-z+F(-z))],
\end{aligned}
$$

where

$$
F(z)=\frac{z \times\left(\sqrt{\widehat{\mathrm{MSE}}\left(\hat{\theta}_{i}^{\mathrm{EB}}-\hat{\theta}_{j}^{\mathrm{EB}}\right)}-\sqrt{g_{1 i}(A)+g_{1 j}(A)}\right)+\hat{\theta}_{i}^{\mathrm{EB}}-\tilde{\theta}_{i}^{B}-\left(\hat{\theta}_{j}^{\mathrm{EB}}-\tilde{\theta}_{j}^{B}\right)}{\sqrt{g_{1 i}(A)+g_{1 j}(A)}}
$$

$g_{1 i}(A)=\frac{A \psi_{i}}{A+\psi_{i}}, \quad g_{1 j}(A)=\frac{A \psi_{j}}{A+\psi_{j}}$. Using the Taylor expansion with an integral remainder term, $\Phi(z+F(z))$ is evaluated as

$$
\begin{aligned}
\Phi(z+F(z)) & =\Phi(z)+F(z) \phi(z)+\frac{1}{2} F^{2}(z) \phi^{\prime}(z)+\frac{1}{2} \int_{z}^{z+F(z)}(z+F(z)-x)^{2} \phi^{\prime \prime}(x) d x \\
& =\Phi(z)+\left[F(z)-\frac{z}{2} F^{2}(z)\right] \phi(z)-\frac{1}{2} \int_{z}^{z+F(z)}(z+F(z)-x)^{2}\left(1-x^{2}\right) \phi(x) d x .
\end{aligned}
$$

Now observe that

$$
\begin{aligned}
E(\Phi(z+F(z)))= & \Phi(z)+E\left[F(z) \phi(z)-\frac{z}{2} F^{2}(z) \phi(z)\right. \\
& \left.-\frac{1}{2} \int_{z}^{z+F(z)}(z+F(z)-x)^{2}\left(1-x^{2}\right) \phi(x) d x\right] .
\end{aligned}
$$

In a similar manner to a one population case, we have

$$
\begin{aligned}
\hat{\theta}_{i}^{\mathrm{EB}}-\tilde{\theta}_{i}^{B} & -\left\{\hat{\theta}_{j}^{\mathrm{EB}}-\tilde{\theta}_{j}^{B}\right\} \\
& =\left(1-\hat{\gamma}_{i}\right) y_{i}+\hat{\gamma}_{i} x_{i}^{\prime} \hat{\beta}-\left(1-\gamma_{i}\right) y_{i}-\gamma_{i} x_{i}^{\prime} \tilde{\beta}- \\
& \left\{\left(1-\hat{\gamma}_{j}\right) y_{j}+\hat{\gamma}_{j} x_{j}^{\prime} \hat{\beta}-\left(1-\gamma_{j}\right) y_{j}-\gamma_{j} x_{j}^{\prime} \tilde{\beta}\right\} \\
& =\left(\gamma_{i}-\hat{\gamma}_{i}\right) r_{i}+\gamma_{i} x_{i}^{\prime}(\hat{\beta}-\tilde{\beta})-\left(\gamma_{j}-\hat{\gamma}_{j}\right) r_{j}-\gamma_{j} x_{j}^{\prime}(\hat{\beta}-\tilde{\beta})
\end{aligned},
$$

where $r_{i}=\left(y_{i}-x_{i}^{\prime} \hat{\beta}\right)$ and $r_{j}=\left(y_{j}-x_{j}^{\prime} \hat{\beta}\right)$. 
Thus $F(z)$ can be decomposed into three components as

$$
\begin{aligned}
F(z) & =\frac{z \times\left(\sqrt{\widehat{\operatorname{MSE}}\left(\hat{\theta}_{i}^{\mathrm{EB}}-\hat{\theta}_{j}^{\mathrm{EB}}\right)}-\sqrt{g_{1 i}(A)+g_{1 j}(A)}\right)}{\sqrt{g_{1 i}(A)+g_{1 j}(A)}} \\
& +\frac{\left(\gamma_{i}-\hat{\gamma}_{i}\right) r_{i}-\left(\gamma_{j}-\hat{\gamma}_{j}\right) r_{j}}{\sqrt{g_{1 i}(A)+g_{1 j}(A)}}+\frac{\gamma_{i} x_{i}^{\prime}(\hat{\beta}-\tilde{\beta})-\gamma_{j} x_{j}^{\prime}(\hat{\beta}-\tilde{\beta})}{\sqrt{g_{1 i}(A)+g_{1 j}(A)}} \\
& =Q_{1}+Q_{2}+Q_{3}, \quad \text { say. }
\end{aligned}
$$

We obtained the expected values of $Q_{1}$ and $Q_{1}^{2}$ given above

$$
\begin{aligned}
E Q_{1} & =\left(g_{1 i}+g_{1 j}\right)^{-1} \frac{z}{2}\left(g_{2 i}(A)+g_{3 i}(A)+g_{2 j}(A)+g_{3 j}(A)\right) \\
& -\left(g_{1 i}+g_{1 j}\right)^{-1} \frac{z}{2}\left(\frac{2 \psi_{i} \psi_{j}}{\left(\hat{A}+\psi_{i}\right)\left(\hat{A}+\psi_{j}\right)} x_{i}^{\prime}\left\{\sum_{i=1}^{m} \frac{x_{i} x_{i}^{\prime}}{\hat{A}+\psi_{i}}\right)^{-1} x_{j}\right) \\
& -\left(g_{1 i}+g_{1 j}\right)^{-2} \frac{z^{2}}{8}\left(\frac{\psi_{i}^{2}}{\left(A+\psi_{i}\right)^{2}}+\frac{\psi_{j}^{2}}{\left(A+\psi_{j}\right)^{2}}\right)^{2} V(\hat{A})+O\left(m^{-3 / 2}\right), \\
E\left(Q_{1}^{2}\right) & =\left(g_{1 i}+g_{1 j}\right)^{-2} z^{2} E\left(\widehat{\operatorname{MSE}}\left(\hat{\theta}_{i}^{\mathrm{EB}}-\hat{\theta}_{j}^{\mathrm{EB}}\right)-\left(g_{1 i}(A)+g_{1 j}(A)\right)\right)^{2} \\
& =\left(\frac{A \psi_{i}}{\left(A+\psi_{i}\right)}+\frac{A \psi_{j}}{\left(A+\psi_{j}\right)}\right)^{-2} z^{2}\left(\frac{\psi_{i}^{2}}{\left(A+\psi_{i}\right)^{2}}+\frac{\psi_{j}^{2}}{\left(A+\psi_{j}\right)^{2}}\right)^{2} V(\hat{A}) \\
& +O\left(m^{-3 / 2}\right) .
\end{aligned}
$$

Let us now evaluate $Q_{2}^{2}$ as

$$
\begin{aligned}
Q_{2}^{2} & =\left(\frac{\left(\gamma_{i}-\hat{\gamma}_{i}\right) r_{i}-\left(\gamma_{j}-\hat{\gamma}_{j}\right) r_{j}}{\sqrt{g_{1 i}(A)+g_{1 j}(A)}}\right)^{2} \\
& =\left(g_{1 i}+g_{1 j}\right)^{-1}\left(\left(\gamma_{i}-\hat{\gamma}_{i}\right) r_{i}-\left(\gamma_{j}-\hat{\gamma}_{j}\right) r_{j}\right)^{2} \\
& =\left(g_{1 i}+g_{1 j}\right)^{-1}\left\{\left(\left(\gamma_{i}-\hat{\gamma}_{i}\right) r_{i}\right)^{2}+\left(\left(\gamma_{j}-\hat{\gamma}_{j}\right) r_{j}\right)^{2}-2\left(\gamma_{i}-\hat{\gamma}_{i}\right) r_{i}\left(\gamma_{j}-\hat{\gamma}_{j}\right) r_{j}\right\} \\
& =Q_{11}+Q_{12}+Q_{13}, \quad \text { Say. }
\end{aligned}
$$

Let us evaluate each term turn by turn as

$$
Q_{11}=\left(g_{1 i}+g_{1 j}\right)^{-1}\left(\gamma_{i}-\hat{\gamma}_{i}\right)^{2}\left(y_{i}-x_{i}^{\prime} \hat{\beta}\right)^{2} \text {. }
$$


Using the Taylor expansion,

$$
\begin{aligned}
Q_{11} & =\left(g_{1 i}+g_{1 j}\right)^{-1}\left(\gamma_{i}^{(1)}(A)\right)^{2}(\hat{A}-A)^{2}\left(y_{i}-x_{i}^{\prime} \tilde{\beta}-x_{i}^{\prime} \tilde{\beta}^{(1)}(A)(\hat{A}-A)\right)^{2} \\
& =\left(g_{1 i}+g_{1 j}\right)^{-1}\left(\gamma_{i}^{(1)}(A)\right)^{2}(\hat{A}-A)^{2}\left(y_{i}-x_{i}^{\prime} \tilde{\beta}\right)^{2} \\
& -2\left(g_{1 i}+g_{1 j}\right)^{-1}\left(\gamma_{i}^{(1)}(A)\right)^{2}(\hat{A}-A)^{2} x_{i}^{\prime} \tilde{\beta}^{(1)}(A)(\hat{A}-A)\left(y_{i}-x_{i}^{\prime} \tilde{\beta}\right) \\
& +\left(g_{1 i}+g_{1 j}\right)^{-1}\left(\gamma_{i}^{(1)}(A)\right)^{2}(\hat{A}-A)^{2}\left(x_{i}^{\prime} \tilde{\beta}^{(1)}(A)(\hat{A}-A)\right)^{2} .
\end{aligned}
$$

We evaluated this part for the case of one population mean, thus

$$
\begin{aligned}
& E Q_{11}=\left(g_{1 i}+g_{1 j}\right)^{-1}\left(\gamma_{i}^{(1)}\right)^{2}\left(A+\psi_{i}\right) E(\hat{A}-A)^{2}, \\
& E Q_{11}=\left(g_{1 i}+g_{1 j}\right)^{-1}\left(\gamma_{i}^{(1)}\right)^{2}\left(A+\psi_{i}\right) V(\hat{A}) .
\end{aligned}
$$

Similarly,

$$
\begin{aligned}
E Q_{12} & =\left(g_{1 i}+g_{1 j}\right)^{-1}\left(\gamma_{j}^{(1)}\right)^{2}\left(A+\psi_{j}\right) V(\hat{A}), \\
Q_{13} & =-2\left(g_{1 i}+g_{1 j}\right)^{-1}\left(\gamma_{i}-\hat{\gamma}_{i}\right)\left(y_{i}-x_{i}^{\prime} \hat{\beta}\right)\left(\gamma_{j}-\hat{\gamma}_{j}\right)\left(r_{j}-x_{j}^{\prime} \hat{\beta}\right) .
\end{aligned}
$$

Using the Taylor expansion,

$$
\begin{aligned}
Q_{13}= & -2\left(g_{1 i}+g_{1 j}\right)^{-1}\left(\gamma_{i}^{(1)}(A)(\hat{A}-A)\left(y_{i}-x_{i}^{\prime} \tilde{\beta}-x_{i}^{\prime} \tilde{\beta}^{(1)}(A)(\hat{A}-A)\right)\right. \\
& \times\left(\gamma_{j}^{(1)}(A)(\hat{A}-A)\left(y_{j}-x_{j}^{\prime} \tilde{\beta}-x_{j}^{\prime} \tilde{\beta}^{(1)}(A)(\hat{A}-A)\right) .\right.
\end{aligned}
$$

Using Stein identity (see equation (8)),

$$
Q_{13}=-2\left(g_{1 i}+g_{1 j}\right)^{-1}\left(A+\psi_{i}\right) \gamma_{i}^{(1)} \frac{\partial \hat{A}}{\partial y_{i}}\left(A+\psi_{j}\right) \gamma_{j}^{(1)} \frac{\partial \hat{A}}{\partial y_{j}}
$$

since $\frac{\partial \hat{A}}{\partial y_{i}}=O_{P}\left(m^{-1}\right)$. Therefore,

$$
\begin{aligned}
Q_{2}^{2} & =Q_{11}+Q_{12}+Q_{13} \\
& =\left(g_{1 i}+g_{1 j}\right)^{-1}\left(\left(\gamma_{i}^{(1)}\right)^{2}\left(A+\psi_{i}\right)+\left(\gamma_{j}^{(1)}\right)^{2}\left(A+\psi_{j}\right)\right) V(\hat{A}) \\
& =\left(\frac{A \psi_{i}}{\left(A+\psi_{i}\right)}+\frac{A \psi_{j}}{\left(A+\psi_{j}\right)}\right)^{-1}\left(\frac{\psi_{i}^{2}}{\left(A+\psi_{i}\right)^{3}}+\frac{\psi_{j}^{2}}{\left(A+\psi_{j}\right)^{3}}\right) V(\hat{A}) .
\end{aligned}
$$


The expected value of $Q_{3}^{2}$ can be given as

$$
\begin{aligned}
E Q_{3}^{2} & =E\left(\frac{\gamma_{i} x_{i}^{\prime}(\hat{\beta}-\tilde{\beta})-\gamma_{j} x_{j}^{\prime}(\hat{\beta}-\tilde{\beta})}{\sqrt{g_{1 i}(A)+g_{1 j}(A)}}\right)^{2} \\
& =\left(g_{1 i}+g_{1 j}\right)^{-1}\left[\gamma_{i}^{2} x_{i}^{\prime}\left\{\sum_{u=1}^{m}\left(A+\psi_{u}\right)^{-1} x_{u} x_{u}^{\prime}\right\}^{-1} x_{i}+\gamma_{j}^{2} x_{i}^{\prime}\left\{\sum_{u=1}^{m}\left(A+\psi_{u}\right)^{-1} x_{u} x_{u}^{\prime}\right\}^{-1} x_{i}\right] \\
& -2\left(g_{1 i}+g_{1 j}\right)^{-1} \gamma_{i} \gamma_{j} x_{i}^{\prime}\left\{\sum_{u=1}^{m}\left(A+\psi_{u}\right)^{-1} x_{u} x_{u}^{\prime}\right\}^{-1} x_{j} \\
& =\left(\frac{A \psi_{i}}{A+\psi_{i}}+\frac{A \psi_{j}}{A+\psi_{j}}\right)^{-1}\left[\frac{\psi_{i}^{2}}{\left(A+\psi_{i}\right)^{2}} x_{i}^{\prime}\left\{\sum_{u=1}^{m}\left(A+\psi_{u}\right)^{-1} x_{u} x_{u}^{\prime}\right\}^{-1} x_{i}\right. \\
& +\frac{\psi_{j}^{2}}{\left(A+\psi_{j}\right)^{2}} x_{i}^{\prime}\left\{\sum_{u=1}^{m}\left(A+\psi_{u}\right)^{-1} x_{u} x_{u}^{\prime}\right\}^{-1} x_{i} \\
& \left.-2 \frac{\psi_{i}}{\left(A+\psi_{i}\right)} \frac{\psi_{j}}{\left(A+\psi_{j}\right)} x_{i}^{\prime}\left\{\sum_{u=1}^{m}\left(A+\psi_{u}\right)^{-1} x_{u} x_{u}^{\prime}\right\}^{-1} x_{j}\right] .
\end{aligned}
$$

The integral and remainder terms can be easily simplified similar to Theorem 1 .

Combination and simplification of all the above results gives as

$$
P\left[\theta_{i}-\theta_{j} \in I^{\mathrm{CEB}}(\hat{A})\right]=2 \Phi(z)-1-\frac{z \phi(z)}{4} \eta+O\left(m^{-3 / 2}\right),
$$

where

$$
\eta=\left(z^{2}+1\right)\left(\frac{A \psi_{i}}{A+\psi_{i}}+\frac{A \psi_{j}}{A+\psi_{j}}\right)^{-2}\left(\frac{\psi_{i}^{2}}{\left(A+\psi_{i}\right)^{2}}+\frac{\psi_{j}^{2}}{\left(A+\psi_{j}\right)^{2}}\right)^{2} V(\hat{A}) .
$$




\section{Appendix D: Graphs and Tables}

PR Method

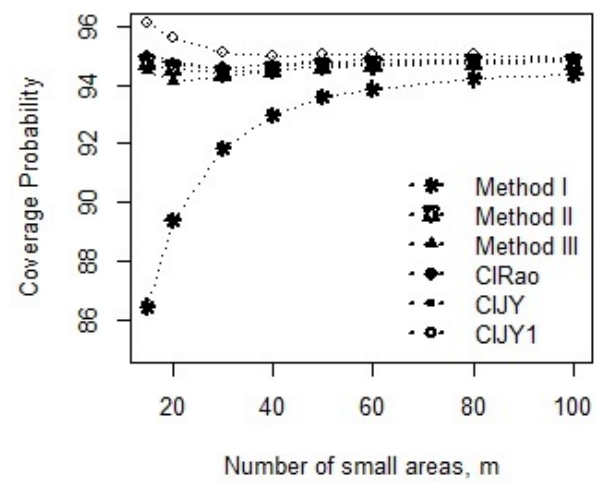

ML Method

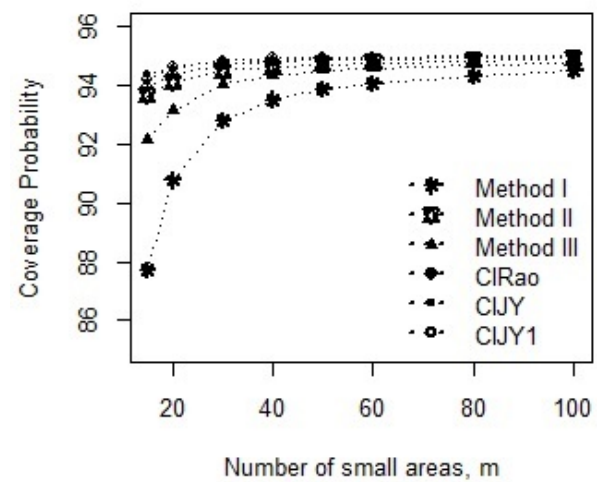

FH Method

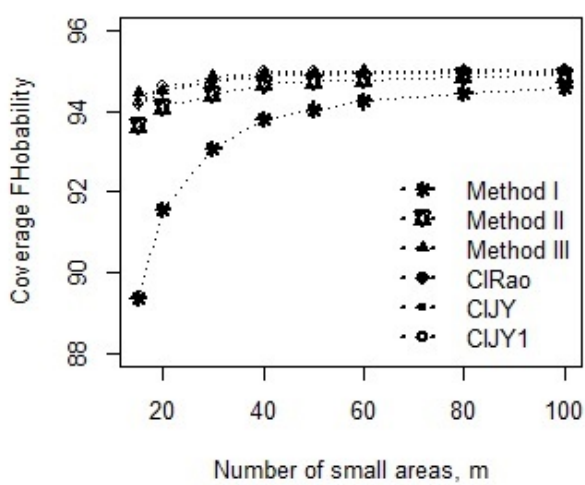

RE Method

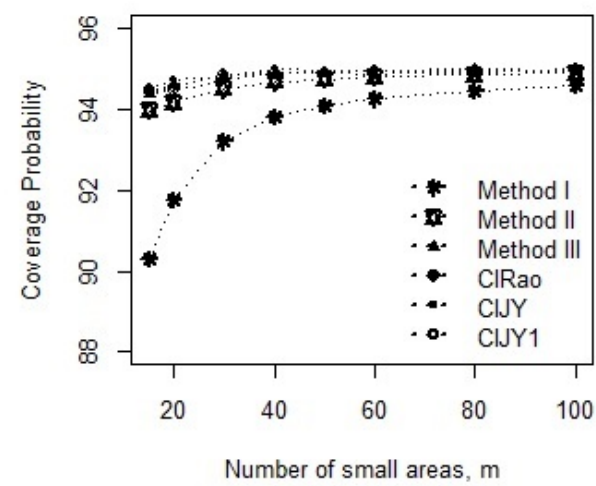

Figure 2: Simulated values of the CPs of Method I, Method II, Method III, CIRao, CIJY and CIJY1 for nominal 95\% CIs for $\psi_{i}$ pattern II. ${ }^{2}$

${ }^{2}$ Meth I, Meth II, Meth III, CIRao, CIJY and CIJY1 referring the CIs given by equations (3.4 - 3.9). Methods of estimating A: PR, Prasad and Rao (1990); ML, maximum likelihood; REML, restricted maximum likelihood; FH, Fay and Herriot (1979). 
PR Method

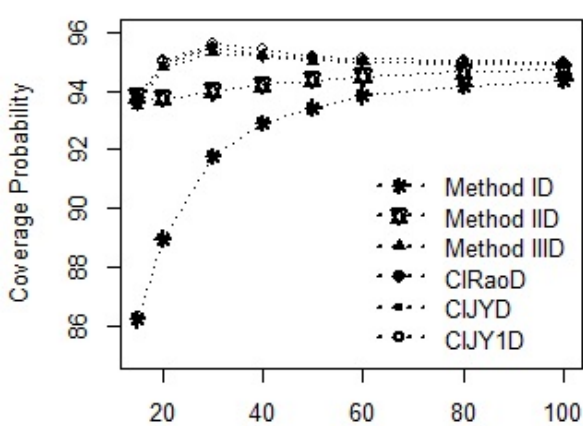

Number of small areas, $m$

\section{Method}

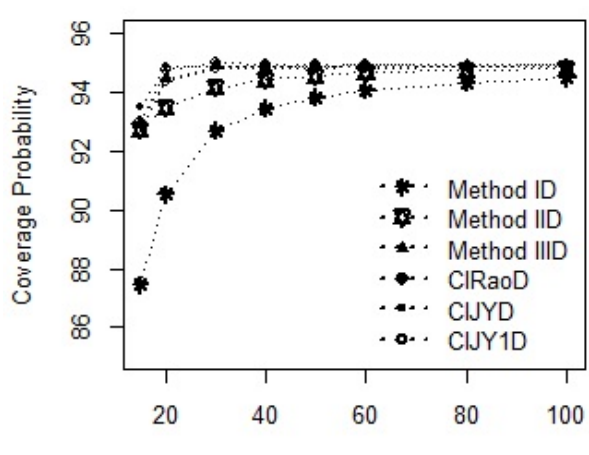

Number of small areas, $m$
FH Method

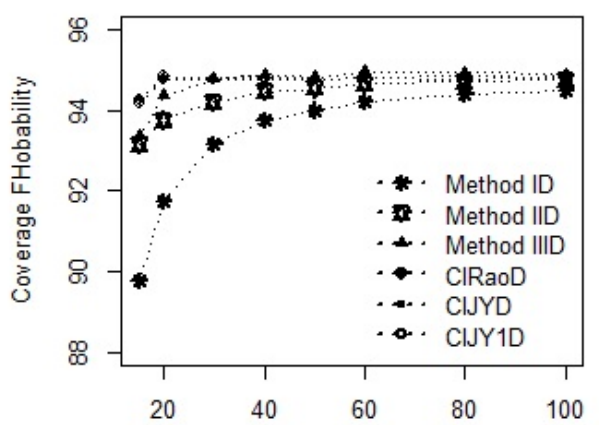

Number of small areas, $m$

\section{RE Method}

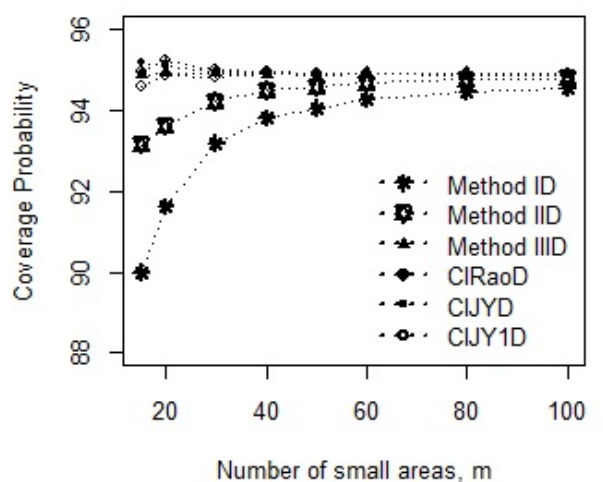

Figure 3: Simulated values of the CPs of Method ID, Method IID, Method IIID, CIRaoD, CIJYD and CIJY1D for nominal 95\% CIs for $\psi_{i}$ pattern $\mathrm{II}^{4}$.

${ }^{4}$ Note that: Method ID, Method IID, Method IIID, CIRaoD, CIJYD and CIJY1D referring the CIs given by equations (3.10 - 3.15). Methods of estimating A: PR, Prasad and Rao (1990); ML, maximum likelihood; REML, restricted maximum likelihood; FH, Fay and Herriot (1979). 


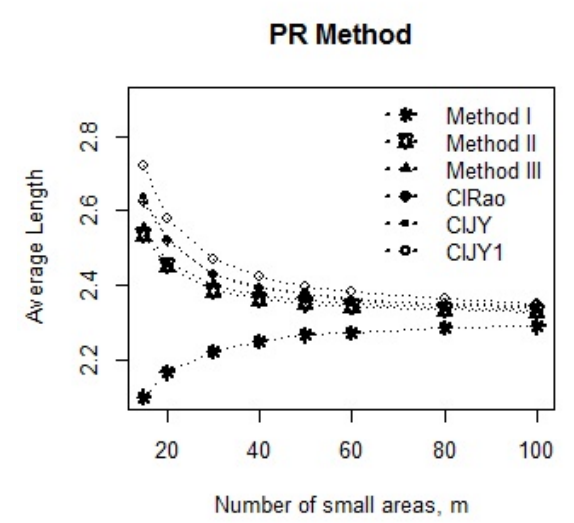

ML Method

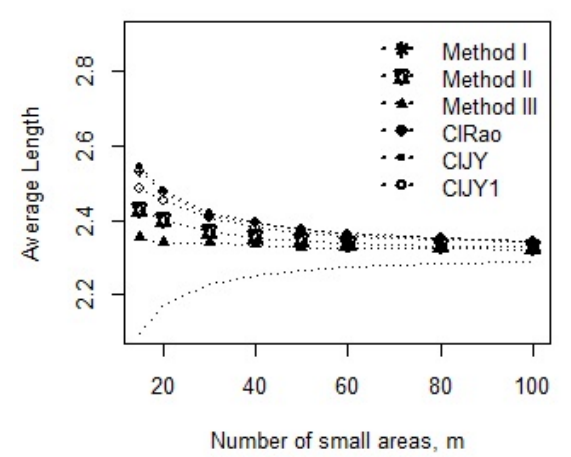

FH Method

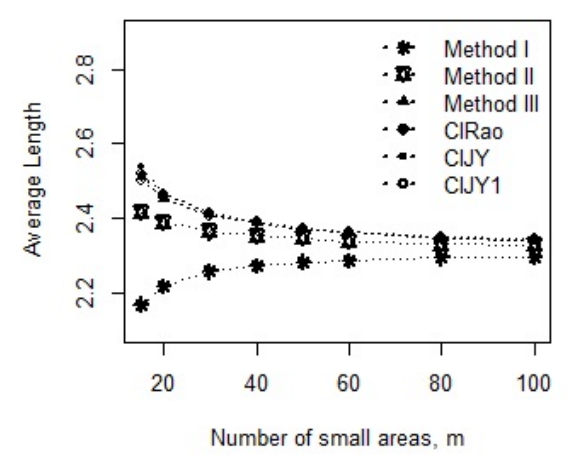

RE Method

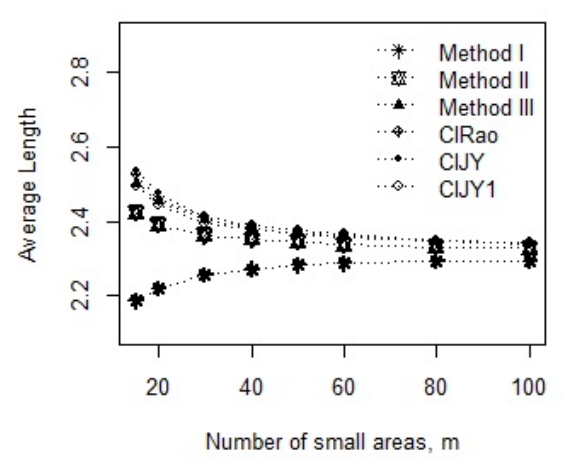

Figure 4: Simulated values of the CPs and ALs of Method I, Method II, Method III, CIRao, CIJY and CIJY1 for nominal 95\% CIs for $\psi_{i}$ pattern II. ${ }^{6}$

\footnotetext{
${ }^{6}$ Meth I, Meth II, Meth III, CIRao, CIJY and CIJY1 referring the CIs given by equations (3.4 - 3.9). Methods of estimating A: PR, Prasad and Rao (1990); ML, maximum likelihood; REML, restricted maximum likelihood; FH, Fay and Herriot (1979).

${ }^{8}$ Note that:

- Method ID, Method IID, Method IIID, CIRaoD, CIJYD and CIJY1D referring the CIs given by equations (3.10 - 3.15).

- Methods of estimating A: PR, Prasad and Rao (1990); ML, maximum likelihood; REML, restricted maximum likelihood; FH, Fay and Herriot (1979).

- Groups: G1, areas 1-3; G2, areas 4-6; G3, areas 7-9; G4, areas 10-12, G5, areas 13-15.
} 


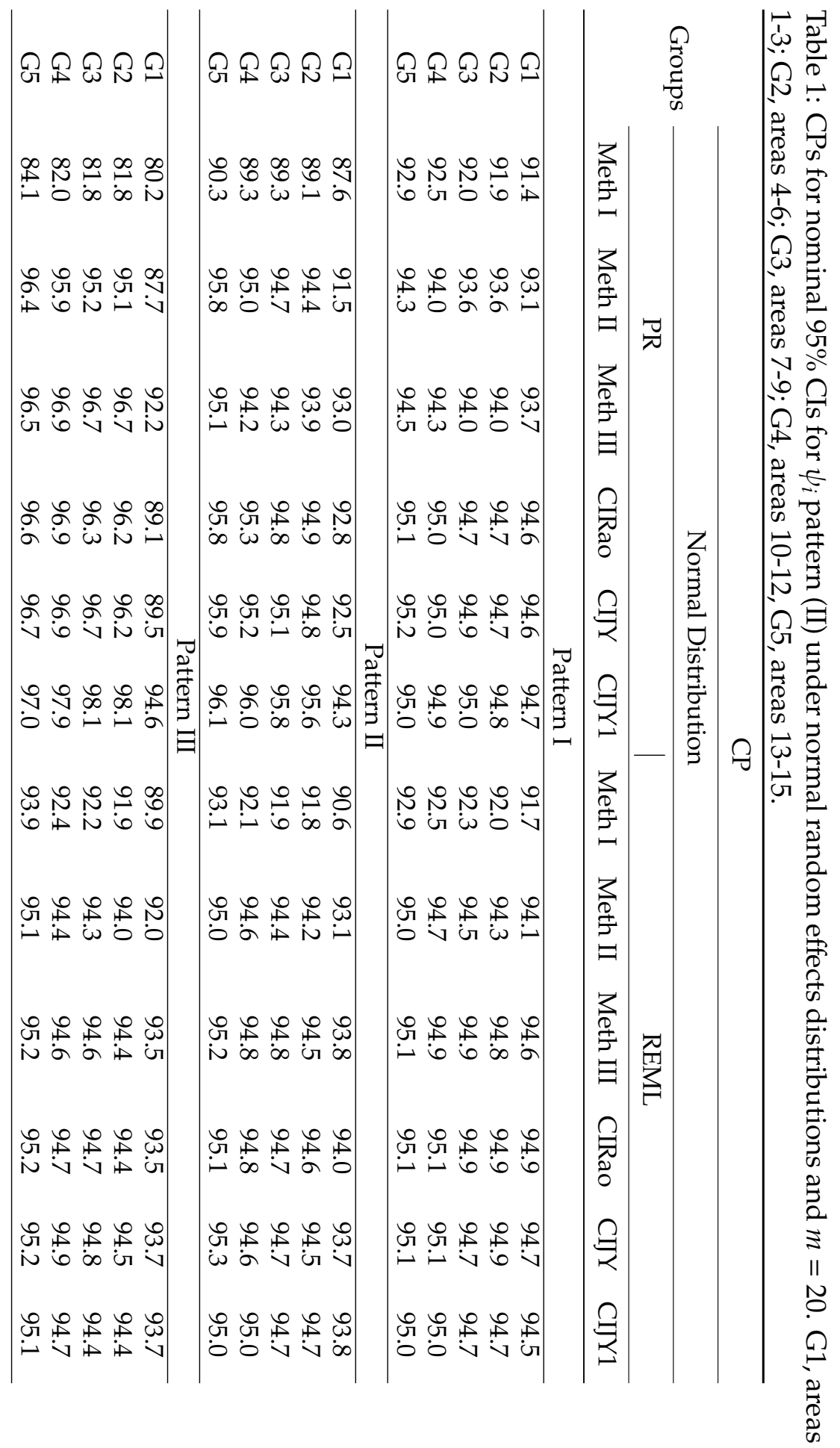




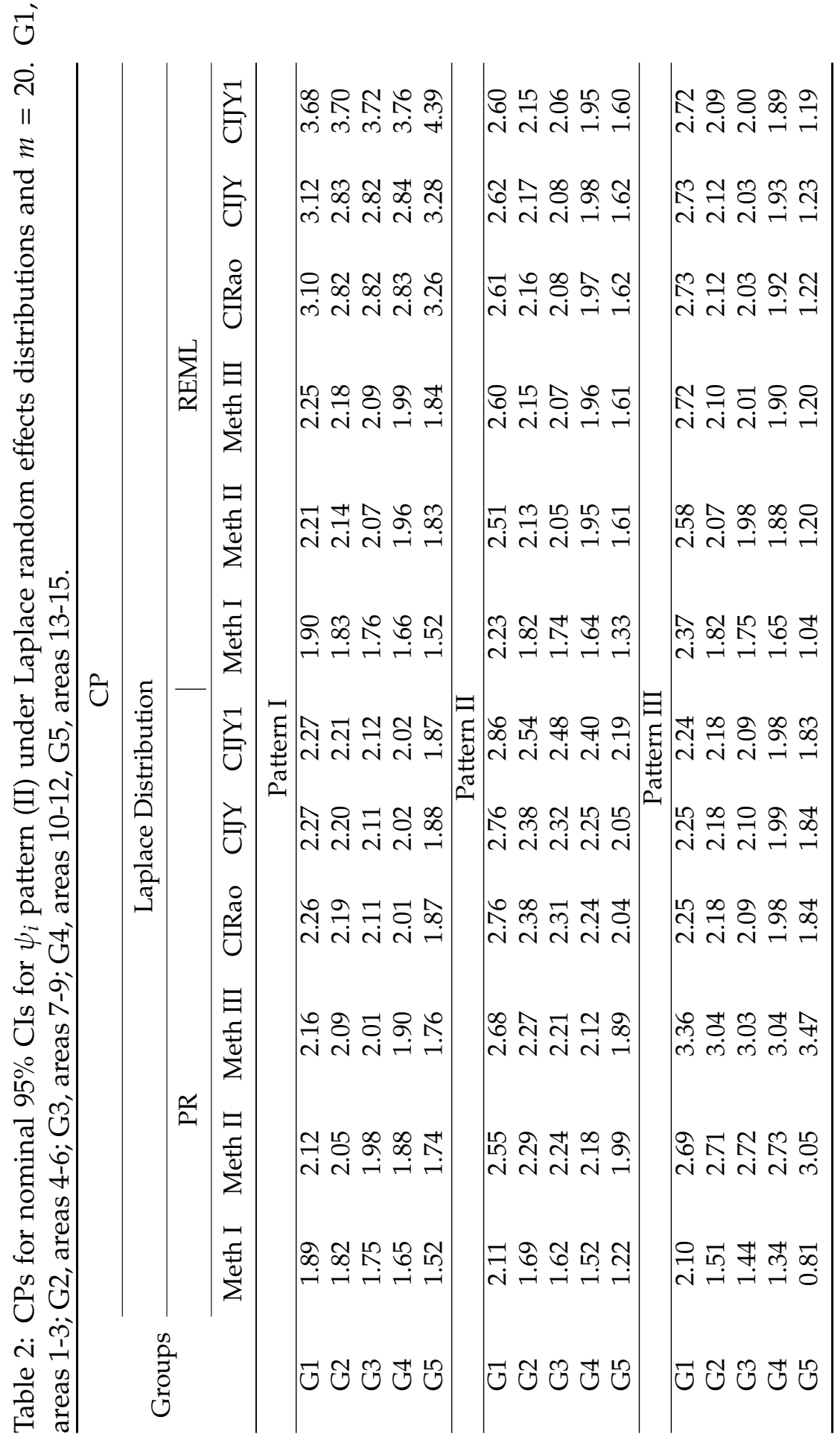




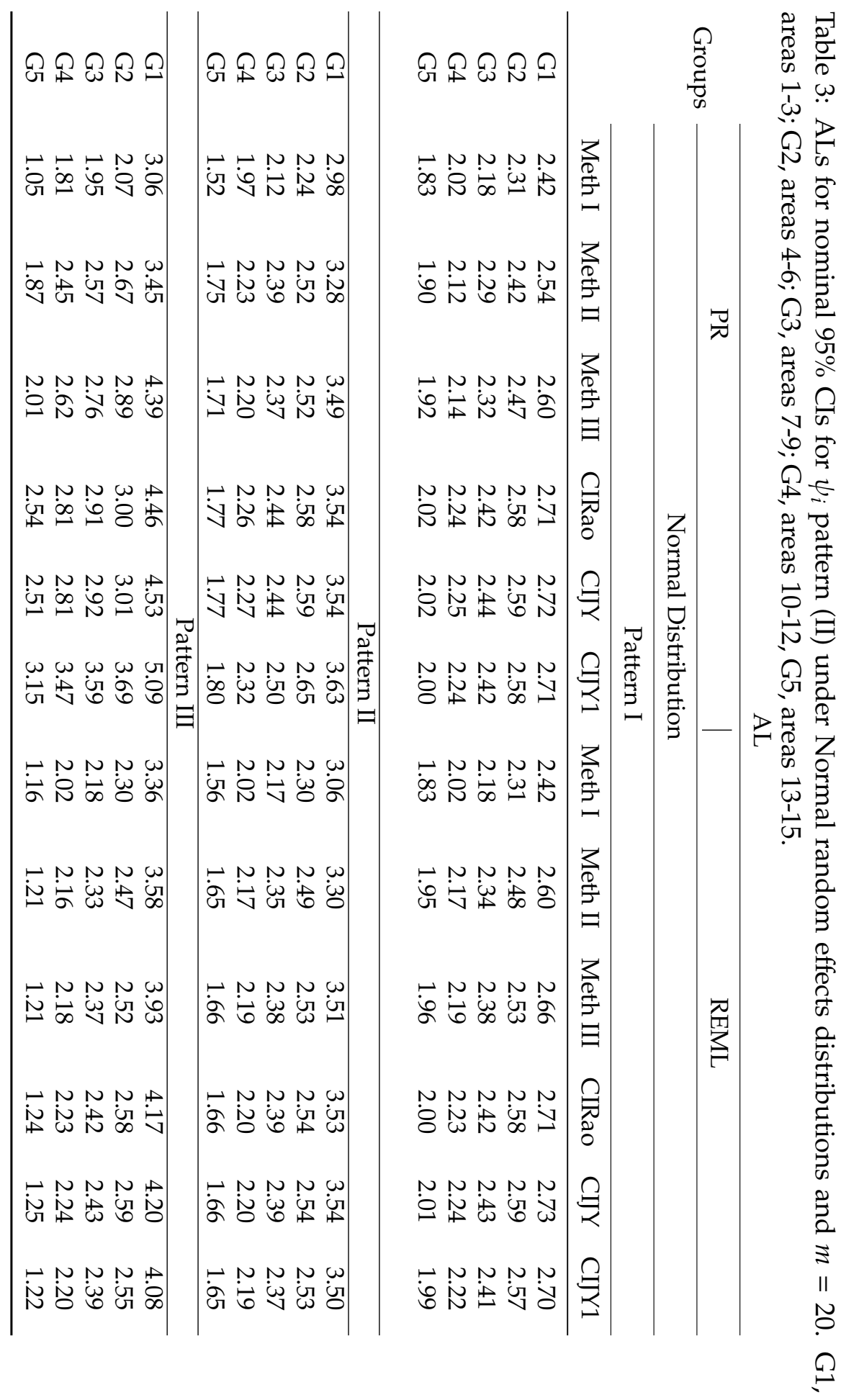




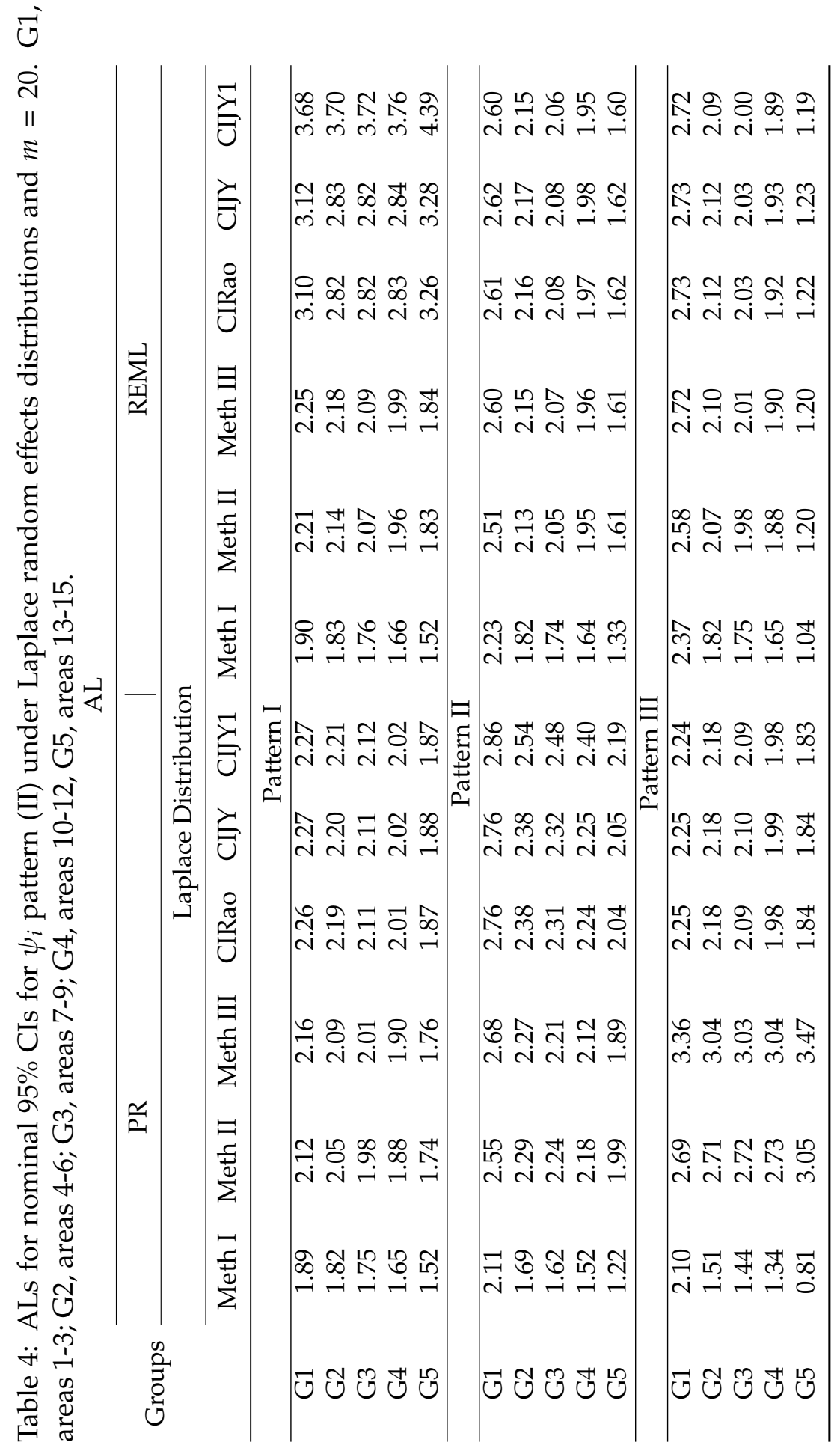




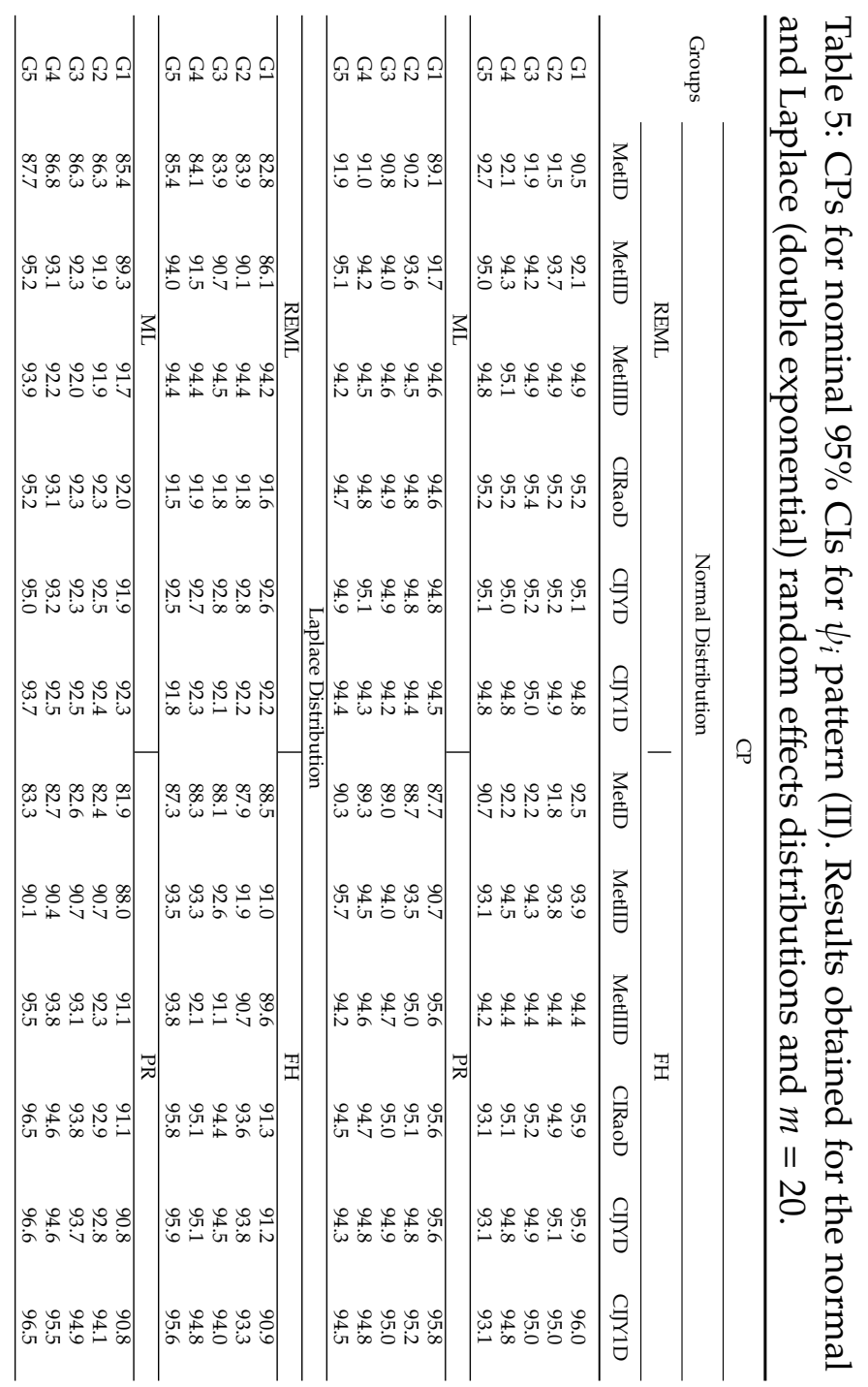




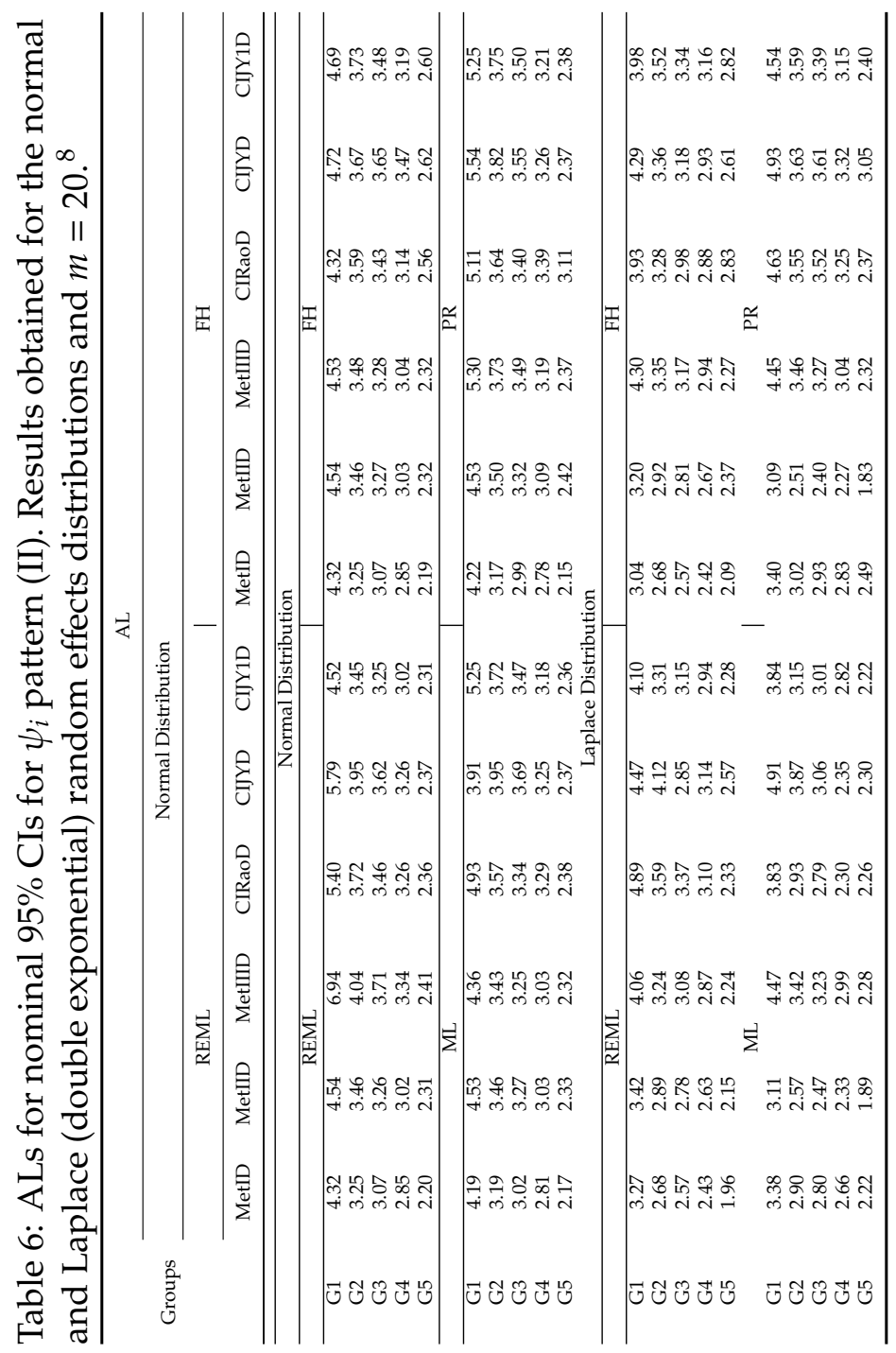

